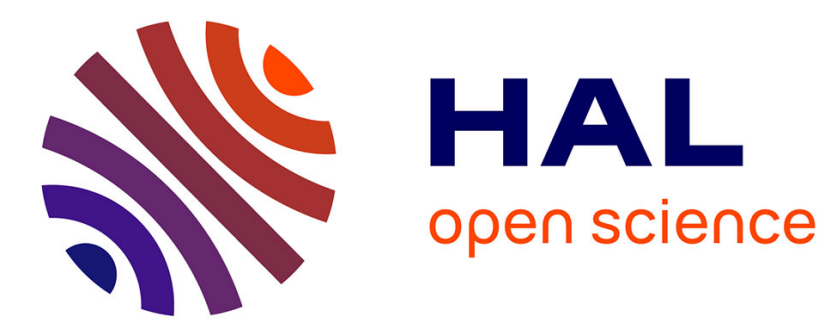

\title{
Parametric instability in a rotating cylinder of gas subject to sinusoidal axial compression. Part 1. Linear theory.
}

\author{
Jean-Philippe Racz, Julian F. Scott
}

\section{To cite this version:}

Jean-Philippe Racz, Julian F. Scott. Parametric instability in a rotating cylinder of gas subject to sinusoidal axial compression. Part 1. Linear theory.. Journal of Fluid Mechanics, 2008, 595, pp.265290. 10.1017/S0022112007009238. hal-00445038

\section{HAL Id: hal-00445038 \\ https://hal.science/hal-00445038}

Submitted on 13 Jan 2010

HAL is a multi-disciplinary open access archive for the deposit and dissemination of scientific research documents, whether they are published or not. The documents may come from teaching and research institutions in France or abroad, or from public or private research centers.
L'archive ouverte pluridisciplinaire $\mathbf{H A L}$, est destinée au dépôt et à la diffusion de documents scientifiques de niveau recherche, publiés ou non, émanant des établissements d'enseignement et de recherche français ou étrangers, des laboratoires publics ou privés. 


\title{
Parametric instability in a rotating cylinder of gas subject to sinusoidal axial compression. Part 1. Linear theory
}

\author{
J.-P. RACZ AND J.F. SCOTT \\ Laboratoire de Mécanique des Fluides et d'Acoustique, ECL, UCBL, INSA, CNRS, \\ 36 avenue Guy de Collongue, 69134 Ecully, France
}

(Received 27 February 2006 and in revised form 19 September 2007)

An analysis is presented of parametric instability in a finite-length rotating cylinder subject to periodic axial compression by small sinusoidal oscillations of one of its ends (the 'piston'). The instability is due to resonant interactions between inertialwave (Kelvin) modes of the cylinder and the oscillatory compression and is resisted by viscosity, acting both through thin boundary layers and throughout the volume, the two mechanisms proving crucial for a satisfactory description. Instability is found to take the form of either a single axisymmetric mode with frequency near to half that of compression, or a pair of non-axisymmetric modes of the same azimuthal and axial orders and oppositely signed frequencies, whose difference is close to the compression frequency. Thus, in the axisymmetric case, instability leads to spontaneous growth of a system of one or more oscillating toroidal vortices encircling the cylinder axis, while the differing frequencies of the two modes of non-axisymmetric instability implies an oscillatory aperiodic flow. The neutral curves giving the threshold for instability are determined for all modes/mode pairs. For a given mode or mode pair, the neutral curve shows a critical piston amplitude dependent on rotational Reynolds number and cylinder aspect ratio, below which instability does not occur, and above which there is instability provided the compression frequency is chosen to lie in a band centred on the exact resonance condition.

\section{Introduction}

The problem studied in this paper is illustrated in figure 1. Gas inside a finite circular cylinder which rotates about its axis is subject to axial sinusoidal compression by oscillations of one of its ends (the 'piston'). The initial motivation for this work (and also that of the related study by Mansour \& Lundgren 1990) was the observed enhancement by swirl of mixing in the cylinders of piston engines. However, the present problem is of more general interest as a new example of parametric instabilities in rotating flows (see the review by Kerswell 2002, who mainly focuses on the most widely studied case, namely the elliptic instability; the introduction and section 6 of that review discuss applications to turbulence dynamics, geophysics, astrophysics, the instability of shear flows and aircraft trailing vortices) which have, as their underlying mechanism, resonant coupling of a pair of inertial-wave modes by an externally applied periodic perturbation. In this context, our aim, through careful analysis and detailed results for the present problem, is to elucidate more clearly the effects of viscosity and (in Part 2, Racz \& Scott 2008) nonlinearity for such instabilities. 


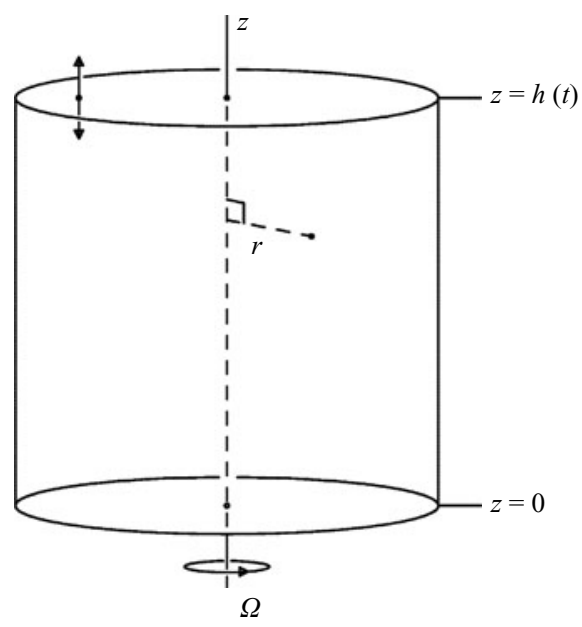

Figure 1. Illustration of the rotating cylinder with an oscillating piston at $z=h(t)$. The $z$-axis and radial coordinate, $r$, are also indicated. A frame of reference rotating with the cylinder is used throughout the paper.

Small externally imposed sinusoidal perturbation of rotating flow, such as the periodic compression of this paper, can have important results owing to resonant interactions with inertial waves. One possibility is direct resonance, in which an inertial mode is driven at or near its natural frequency, leading to modal growth until either viscosity or nonlinearity intervenes (see e.g. Fultz 1959; Baines 1967; Aldridge \& Toomre 1969; Gans 1970; McEwan 1970; Stergiopoulos \& Aldridge 1982; Rieutord 1991; Manasseh 1994; Kobine 1995). Although direct resonance induces transient modal growth, this growth is not symptomatic of an instability. On the other hand, another type of resonance, and the one that concerns us in this paper, involves interactions of an oscillatory external perturbation and two inertial modes. One of the modes, of frequency $\omega$, interacts with the external perturbation, frequency $\omega_{0}$, producing interaction frequencies $\omega \pm \omega_{0}$, one of which drives the other mode at its natural frequency if $\omega_{0}$ coincides with the difference of the two modal frequencies (or nearly so: the width of the band of resonant $\omega_{0}$ being proportional to the external perturbation amplitude, supposed small). The resulting parametric resonance can lead to instability via growth of the pair of inertial modes.

The elliptic instability, which has been the subject of considerable work (see Kerswell 2002 and references therein; Eloy, Le Gal \& Le Dizés 2003), results from such a resonance (Waleffe 1990; Gledzer \& Ponomarev 1992), as does the instability which is the subject of this paper. Elliptic flow with small eccentricity is a small perturbation of solid-body rotation and, in the rotating frame of reference used throughout this paper, consists of a sinusoidal flow with frequency $\omega_{0}=2 \Omega, \Omega$ being the rotational angular velocity. Thus, parametric resonance of elliptic flow involves two inertial modes whose frequency difference is close to $2 \Omega$. Although the external perturbation associated with elliptic flow has different spatial structure from that induced by piston oscillations in our problem, and so couples different pairs of modes, the two problems have many features in common, both in the linear theory of this paper and the weakly nonlinear theory of Part 2. Note that, whereas the external perturbation frequency, $2 \Omega$, of elliptic flow has a fixed relation to the rotation rate, that of the piston in the present problem can be chosen at will. Other examples are provided by precession of 
a rotating container of fluid (see e.g. Kerswell 1993, 2002, §5.1; Mahalov 1993), and multipolar instabilities of higher order than the elliptic one (Eloy \& Le Dizés 2001; Eloy et al. 2003).

Most theoretical studies have been inviscid. Although inviscid theory permits an understanding of the origin of the instability, no critical perturbation amplitude is predicted and the flow is unstable to modes of unboundedly small wavelengths. Viscous damping of inertial modes resists the instability, leading to a threshold amplitude of external perturbation, below which instability does not occur, as well as eliminating unstable growth of sufficiently short wavelengths. Furthermore, as we shall see in our problem, the stability criterion obtained by taking the zero viscosity limit does not in general coincide with that derived from inviscid theory, while neglect of viscosity leads to singular behaviour in the weakly nonlinear regime, as will be seen in Part 2. For these reasons, a satisfactory description of instability must allow for viscosity, as we shall do.

In a finite container, such as the cylinder considered in this paper, there are two types of viscous damping: that due to the boundary layers; and volumetric damping due to viscous action throughout the rest of the container. An external perturbation is necessary for instability and, given small amplitude, the viscosity should also be small in order not to kill the instability altogether. Smallness of viscosity is expressed via a large rotational Reynolds number, $R e=2 \Omega a^{2} / v$ (used here in preference to the Ekman number, $E=R e^{-1}$, which is perhaps more traditional in the theory of rotating flows), where $a$ is a length scale characterizing the size of the container and which is taken as the cylinder radius in our case. In the classical theory of weakly viscous modes in a rigidly rotating container (Greenspan 1969), the only damping mechanism allowed for is due to the boundary layers, of thickness $O\left(R e^{-1 / 2}\right)$. Ekman pumping by the boundary layers leads to weak $\left(O\left(R e^{-1 / 2}\right)\right)$ secondary flow in the interior of the container and a modal damping rate of order $R e^{-1 / 2} \Omega$, inducing modal decay on a time scale which is longer, by a factor of $R e^{1 / 2}$, than that of the modal oscillations.

Boundary-layer damping of modes in a finite container may be contrasted with the case of plane inertial waves in an unbounded fluid, for which viscous damping acts throughout the flow and is proportional to the product of $R e^{-1}$ and the squared wavenumber, increasing rapidly at smaller wavelengths. For the low-order modes of a container, whose wavelengths are comparable with the container size, the volumetric damping rate is $O\left(R e^{-1}\right)$, asymptotically small compared with that, $O\left(R e^{-1 / 2}\right)$, arising from the layers, which is why the volumetric component is neglected in the classical theory. On the other hand, although boundary-layer damping varies from mode to mode, it remains $O\left(R e^{-1 / 2}\right)$ for the higher-order modes, whereas volumetric damping increases and the two become comparable for modes of wavelengths $O\left(R e^{-1 / 4}\right)$, with volumetric viscous effects dominating at still shorter wavelengths. Thus, a uniform description, capable of handling modes of all orders, should include both, allowing a continuous passage from the low-order modes, through those for which volumetric damping becomes significant, to the volumetrically dominated damping of the highestorder modes. As we shall see, the total modal damping in a cylinder can be expressed as the sum of boundary-layer and volumetric contributions, both of which are crucial for the treatment of the instabilities we wish to study. In particular, the volumetric component is required to avoid the instability to high-order modes of unboundedly short wavelengths noted earlier. Boundary-layer damping is also required, otherwise the damping rates of the low-order modes, which are the least damped and hence the first to become unstable as the external perturbation amplitude is increased, 
are grossly underestimated. That is, boundary-layer damping controls the threshold external perturbation amplitude for instability.

As noted earlier, the subject of this paper is flow inside a finite cylinder of radius $a$, rotating (along with its endwalls) at angular velocity $\Omega$ about its axis, one of whose ends (the piston) executes small sinusoidal oscillations of frequency $\omega_{0}$ (comparable with $\Omega$ ) and hence subjects the gas inside the cylinder to periodic compression (figure 1). The basic flow whose instabilities are studied has the defining properties that it is axisymmetric and periodic with the piston period. In the limit of zero viscosity and in the frame of reference rotating with the cylinder used throughout the paper, this flow consists of simple periodic compression of the gas column in which fluid particles oscillate axially with displacements which vary linearly with the axial coordinate $z$, from zero at the fixed end $z=0$ to that imposed by the piston at $z=h(t)$. The basic flow considered in this paper is the flow just described, modified by the addition of small viscosity. Mansour \& Lundgren (1990) undertook a linearized numerical study of inviscid rotating flow between two infinite parallel plates, the oscillations of one of which induce periodic compression, a limiting case of the present problem in which viscosity is neglected and the cylinder radius is infinite. They determined the unstable bands of piston frequency as a function of the piston amplitude for a given inertial-wave mode (including bands arising from higher-order parametric resonances which are killed off by viscous damping given the Reynolds-number scaling of the present paper, chosen so that viscosity affects the primary resonance). Finiteness of the cylinder in our case leads to discrete modes, rather than the continuum of the infinite problem, while, as discussed earlier, viscosity allows a more satisfactory description of the instability with a threshold piston amplitude for each mode.

The paper is organized as follows. The starting point in $\S 2$ is the low-Mach-number limiting form of the compressible Navier-Stokes equations. Following transformation of the $z$-coordinate to render the cylinder geometry fixed, the basic flow is introduced and equations for the perturbation are derived (to avoid confusion, note that hereinafter, the term perturbation no longer refers to the externally imposed periodic oscillations, as it did earlier, but instead to perturbations of the basic flow, which already includes the external perturbation). In $\S 3$, the inertial modes are introduced, the velocity perturbation is expanded in terms of the modal eigenfunctions and exact evolution equations for the modal amplitudes derived. Asymptotics based on small sinusoidal piston amplitude is used in $\S 4$ to derive amplitude equations for pairs of parametrically resonant modes which form the basis of the stability analysis and results of $\S 5$.

\section{Formulation}

\subsection{Low-Mach-number equations}

Our starting point is the low-Mach-number limiting form of the compressible NavierStokes equations for a gas with two thermodynamic degrees of freedom in a frame of reference rotating with the cylinder:

$$
\begin{aligned}
\nabla \cdot \boldsymbol{v} & =-\frac{1}{\rho} \frac{\mathrm{D} \rho}{\mathrm{D} t}, \\
\rho \frac{\mathrm{D} \boldsymbol{v}}{\mathrm{D} t} & =-\nabla p^{\prime}+\nabla \cdot \tau-2 \rho \boldsymbol{\Omega} \times \boldsymbol{v}-\rho \boldsymbol{\Omega} \times(\boldsymbol{\Omega} \times \boldsymbol{x}), \\
\rho T \frac{\mathrm{D} s}{\mathrm{D} t} & =\nabla \cdot(k \nabla T),
\end{aligned}
$$


where $\boldsymbol{v}, \rho, \boldsymbol{\tau}, T, s$ and $k$ are the fluid velocity, density, viscous stress tensor, absolute temperature, entropy per unit mass and thermal conductivity, $\boldsymbol{\Omega}$ is the rotational angular velocity of the cylinder and $\mathrm{D} / \mathrm{D} t=\partial / \partial t+\boldsymbol{v} \cdot \nabla$ a material derivative. The differences between the low- $M$ and full compressible Navier-Stokes equations are that: (i) viscous dissipation is neglected in (2.3), and (ii) the thermodynamically determined quantities $\rho(p, s), T(p, s)$ in $(2.1)-(2.3)$, as well as $k(p, s)$ and the coefficients of viscosity implicit in the Newtonian expression for $\boldsymbol{\tau}$, are to be evaluated at uniform pressure $\bar{p}(t)$. The total fluid pressure is $p=\bar{p}(t)+p^{\prime}$, where $p^{\prime}$ represents small $\left(O\left(M^{2}\right)\right)$ departures from uniformity and appears only via the pressure gradient term in $(2.2)$.

For simplicity, adiabatic thermal boundary conditions $(\partial T / \partial n=0)$ and initially uniform entropy $s_{0}$ are supposed. The solution of (2.3) is then $s=s_{0}$, since the corresponding $T=T\left(\bar{p}, s_{0}\right)$ is uniform. Indeed, all thermodynamically determined quantities are uniform, taking their values at pressure $\bar{p}(t)$ and entropy $s_{0}$, but evolving with time owing to variation of $\bar{p}(t)$. In particular, the density is uniform and inversely proportional to the cylinder height $h(t)$, thanks to overall mass conservation. In consequence, (2.1) becomes

$$
\nabla \cdot v=\frac{1}{h} \frac{\mathrm{d} h}{\mathrm{~d} t},
$$

showing that $\nabla \cdot \boldsymbol{v}$ is uniform. Using this fact, the Newtonian expression for $\boldsymbol{\tau}$ and uniformity of the coefficients of viscosity, (2.2) yields

$$
\frac{\mathrm{D} \boldsymbol{v}}{\mathrm{D} t}=-\nabla \pi-2 \boldsymbol{\Omega} \times \boldsymbol{v}+v(t) \nabla^{2} \boldsymbol{v},
$$

where $v(t)$ is the kinematic viscosity, $\pi=p^{\prime} / \rho-\Omega^{2} r^{2} / 2$ is a pressure variable incorporating the centrifugal force and $r$ is the distance from the rotation axis. Because the gas has only two thermodynamic degrees of freedom, the quantity $v$ in (2.5) can, in principle, be determined from the entropy $s_{0}$ and density $\rho(t)$, where the latter is obtained by dividing the known total mass of gas by the cylinder volume.

From here on, we non-dimensionalize using the cylinder radius $a$ and time scale $(2 \Omega)^{-1}$. Thus, (2.4) and (2.5) become

$$
\begin{aligned}
\nabla \cdot v & =\frac{1}{h} \frac{\mathrm{d} h}{\mathrm{~d} t}, \\
\frac{\mathrm{D} \boldsymbol{v}}{\mathrm{D} t} & =-\nabla \pi-\boldsymbol{e}_{\mathrm{z}} \times \boldsymbol{v}+R e^{-1} \nabla^{2} \boldsymbol{v},
\end{aligned}
$$

where $\boldsymbol{e}_{\mathrm{z}}$ is a unit vector in the direction of $\boldsymbol{\Omega}$ (the $z$-direction) and $\operatorname{Re}(t)=2 \Omega a^{2} / v$ is a Reynolds number which inherits the time variations of $v$. Note that, hereinafter, $h(t)$ is non-dimensional, equal to the ratio, $h / a$, of dimensional cylinder height and radius. The boundary conditions are $v=0$ on the sides $(r=1)$ and bottom end $(z=0)$ of the cylinder, while $\boldsymbol{v}=(\mathrm{d} h / \mathrm{d} t) \boldsymbol{e}_{z}$ at the top end $(z=h(t))$. Equations (2.6) and (2.7), together with these boundary conditions, govern the flow.

\subsection{Transformation to fixed geometry}

Writing $x$ and $y$ for the remaining coordinates of a Cartesian system $x, y, z$, it is convenient to transform to fixed cylinder geometry via the new spatial coordinates

$$
X=x, \quad Y=y, \quad Z=\frac{h_{0}}{h(t)} z,
$$


which collectively form a new position vector $X=(X, Y, Z)$. The top end of the cylinder is transformed to the fixed position $Z=h_{0}$, where $h_{0}$ is a reference height, arbitrary for the moment, but which will be taken as the time-average of $h(t)$ when we later specialize to periodic piston motion. New velocity and pressure variables, $\boldsymbol{V}$ and $\Pi$, are introduced according to

$$
V_{X}=v_{x}, \quad V_{Y}=v_{y}, \quad V_{Z}=\frac{h_{0}}{h}\left(v_{z}-\frac{z}{h} \frac{\mathrm{d} h}{\mathrm{~d} t}\right), \quad \Pi=\pi+\frac{1}{2 h} \frac{\mathrm{d}^{2} h}{\mathrm{~d} t^{2}} z^{2} .
$$

With these transformations, the governing equations, (2.6) and (2.7), become

$$
\begin{aligned}
\nabla \cdot \boldsymbol{V}= & 0 \\
\frac{\partial \boldsymbol{V}}{\partial t}+\boldsymbol{e}_{Z} \times \boldsymbol{V}+\nabla \Pi= & R e^{-1} \mathscr{D} \boldsymbol{V}-\boldsymbol{V} \cdot \nabla \boldsymbol{V} \\
& +\boldsymbol{e}_{Z}\left[\frac{\partial}{\partial t}+\boldsymbol{V} \cdot \nabla-\operatorname{Re}^{-1} \mathscr{D}\right]\left(1-\left(\frac{h}{h_{0}}\right)^{2}\right) V_{Z},
\end{aligned}
$$

where, hereinafter, $\nabla$ denotes the operator

$$
\nabla=\left(\frac{\partial}{\partial X}, \frac{\partial}{\partial Y}, \frac{\partial}{\partial Z}\right)
$$

and

$$
\mathscr{D}=\frac{\partial^{2}}{\partial X^{2}}+\frac{\partial^{2}}{\partial Y^{2}}+\left(\frac{h_{0}}{h}\right)^{2} \frac{\partial^{2}}{\partial Z^{2}}
$$

is the Laplacian operator expressed in terms of the new coordinates. The boundary conditions take the form

$$
\begin{aligned}
& \boldsymbol{V}=0, \quad Z=0, \quad h_{0} \\
& \boldsymbol{V}=-\frac{Z}{h} \frac{\mathrm{d} h}{\mathrm{~d} t} \boldsymbol{e}_{Z}, \quad r=1 .
\end{aligned}
$$

\subsection{Basic flow and perturbation}

A particular solution of (2.10) and (2.11) is $\boldsymbol{V}=0, \Pi=0$, which yields

$$
v_{x}=v_{y}=0, \quad v_{z}=\frac{\mathrm{d} h}{\mathrm{~d} t} \frac{z}{h}, \quad \pi=-\frac{\mathrm{d}^{2} h}{\mathrm{~d} t^{2}} \frac{z^{2}}{2 h},
$$

when expressed in terms of the original variables. Of the boundary conditions, this flow fails to satisfy only the tangential component of the no-slip condition on $r=1$ and is thus a solution of the inviscid problem. It corresponds to a simple motion in which fluid particles oscillate axially in response to the piston motion, with a single particle maintaining constant values of $x, y$ and $z / h$. This, or rather its viscous equivalent, forms the basic flow whose instability is the subject of this paper.

From here on, we assume time-periodic piston motion. The inviscid flow (2.15) is then periodic with the piston period and it seems reasonable to suppose that such a flow also exists in the presence of viscosity. It is this property of periodicity with the same period as the piston which defines the basic flow and distinguishes it from the flows arising from its instability. However, we know of no exact analytical expression for the basic flow with both viscosity and piston motion. At the small piston amplitudes and high Reynolds numbers to which we specialize later in this paper, we expect viscosity to induce a thin boundary layer on $r=1$ and more complicated flows near the cylinder corners, with Ekman pumping by the boundary 
layer and corner regions inducing secondary flows in the interior of the cylinder. Denoting the basic flow by $\boldsymbol{V}=\boldsymbol{U}(\boldsymbol{X}, t), \Pi=\Lambda(\boldsymbol{X}, t), \boldsymbol{U}$ is the difference between the basic-flow velocity field with and without viscosity and should therefore consist of the components just mentioned. Note that $\boldsymbol{U}(\boldsymbol{X}, t)$ and $\Lambda(\boldsymbol{X}, t)$ are not determined in this study (see Duguet, Scott \& Le Penven (2005) for numerical results for particular parameter values) because, as we shall see, they contribute only at asymptotic orders above those to which we will work.

Introducing a perturbation to the basic flow:

$$
\boldsymbol{V}=\boldsymbol{U}+\boldsymbol{u}, \quad \Pi=\Lambda+\lambda,
$$

where $\boldsymbol{u}$ and $\lambda$ represent the perturbation, whose governing equations are obtained by subtraction of (2.10) and (2.11) applied to the basic flow from the same equations for the perturbed flow (2.16). Thus,

$$
\begin{aligned}
\nabla \cdot \boldsymbol{u}= & 0 \\
\frac{\partial \boldsymbol{u}}{\partial t}+\boldsymbol{e}_{Z} \times \boldsymbol{u}+\nabla \lambda= & \boldsymbol{e}_{Z} \frac{\partial}{\partial t}\left(\left(1-\left(\frac{h}{h_{0}}\right)^{2}\right) u_{Z}\right)+R e^{-1} \mathscr{D}\left(\boldsymbol{u}_{\perp}+\boldsymbol{e}_{Z}\left(\frac{h}{h_{0}}\right)^{2} u_{Z}\right) \\
& -\boldsymbol{u} \cdot \nabla\left(\boldsymbol{U}_{\perp}+\boldsymbol{e}_{Z}\left(\frac{h}{h_{0}}\right)^{2} U_{Z}\right)-\boldsymbol{V} \cdot \nabla\left(\boldsymbol{u}_{\perp}+\boldsymbol{e}_{Z}\left(\frac{h}{h_{0}}\right)^{2} u_{Z}\right),
\end{aligned}
$$

where $\boldsymbol{U}_{\perp}=\left(U_{X}, U_{Y}, 0\right)$ and $\boldsymbol{u}_{\perp}=\left(u_{X}, u_{Y}, 0\right)$ denote the transverse parts of $\boldsymbol{U}$ and $\boldsymbol{u}$. The boundary conditions on the perturbation are $\boldsymbol{u}=0$ on all boundaries.

Note that in the absence of piston motion ( $h=h_{0}$ constant), the basic flow is $\boldsymbol{v}=0$, corresponding to solid-body rotation in a non-rotating frame. It is well-known that solid-body rotation is unconditionally stable, i.e. any perturbation will eventually decay to zero. It follows that piston motion is necessary for instability.

\section{Inertial modes and the exact modal amplitude equations}

\subsection{Inertial modes}

Kelvin (1880; see also Greenspan 1969, §§2.7, 2.15) derived the inertial-wave eigenmodes of a rotating cylinder which will be used as a basis set to expand the velocity perturbation. These modes are solutions of the inviscid linear problem without piston motion of the form

$$
\boldsymbol{u}=\boldsymbol{u}^{(\mu)}(\boldsymbol{X}) \exp \left(-\mathrm{i} \omega^{(\mu)} t\right), \quad \lambda=\lambda^{(\mu)}(\boldsymbol{X}) \exp \left(-\mathrm{i} \omega^{(\mu)} t\right),
$$

where $\mu$ is a modal index and the modal frequencies, $\omega^{(\mu)}$, and spatial factors, $\boldsymbol{u}^{(\mu)}(\boldsymbol{X})$ and $\lambda^{(\mu)}(\boldsymbol{X})$, are the eigenvalues and eigenfunctions of the problem

$$
\begin{gathered}
\nabla \cdot \boldsymbol{u}^{(\mu)}=0, \\
-\mathrm{i} \omega^{(\mu)} \boldsymbol{u}^{(\mu)}+\boldsymbol{e}_{Z} \times \boldsymbol{u}^{(\mu)}+\nabla \lambda^{(\mu)}=0,
\end{gathered}
$$

with the inviscid boundary conditions that the normal component of $\boldsymbol{u}^{(\mu)}$ be zero at the walls $r=1$ and $Z=0, h_{0}$. The modal frequencies have the well-known properties (Greenspan 1969) that they are real (implying purely oscillatory modes) and densely fill the range $\left|\omega^{(\mu)}\right|<1$ (dimensional frequencies less than $2 \Omega$ ). Note that the eigenvalue problem, and hence $\omega^{(\mu)}, \boldsymbol{u}^{(\mu)}$ and $\lambda^{(\mu)}$, depends on only one parameter, namely the cylinder aspect ratio $h_{0}$. 
(a)

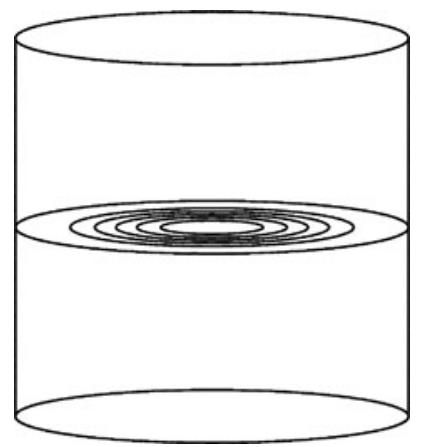

(b)

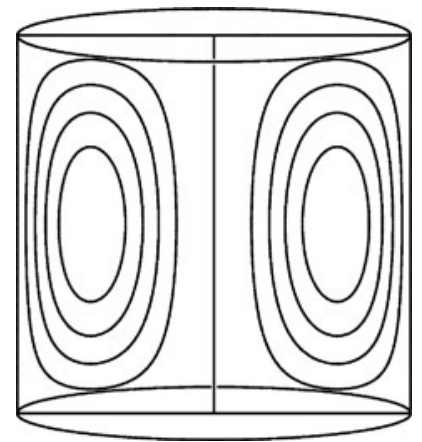

(c)

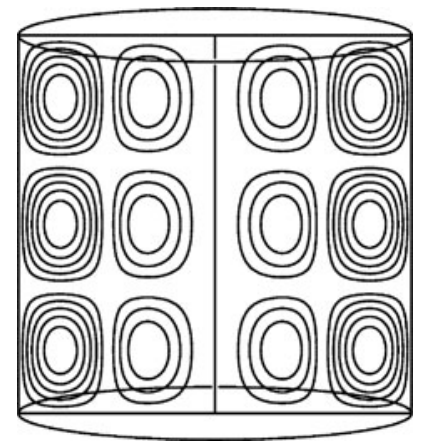

FIGURE 2. (a) Streamlines (circles) in the plane $Z=h_{0} / 2$ for a mode of the axisymmetric, geostrophic family $n=m=0$. $(b, c)$ Streamlines in a plane through the cylinder axis for the two axisymmetric inertial modes defined by: $(b) m=1$ and the lowest positive zero of $J_{0}^{\prime}(k)$, and $(c) m=3$ and the second positive zero of $J_{0}^{\prime}(k)$. The larger $m$ and the zero of $J_{0}^{\prime}(k)$, the more toroidal vortices constitute the mode. As well as the $u_{r}$ and $u_{Z}$ velocity components illustrated here, the modes have a $u_{\theta}$ component whose oscillations are $\pi / 2$ out of phase.

Modes may be classified into families indexed by a pair of integers: $n$, which can take any integer value, and $m \geqslant 0$. Using cylindrical coordinates $r, \theta, Z$ (with $\theta$ measured positive in the sense of the cylinder rotation), modes of the family $n, m$ have eigenfunctions of the form

$$
\left[\begin{array}{c}
u_{r}^{(\mu)} \\
u_{\theta}^{(\mu)} \\
u_{Z}^{(\mu)} \\
\lambda^{(\mu)}
\end{array}\right]=e^{\mathrm{in} \theta}\left[\begin{array}{c}
\mathrm{i} \cos \frac{m \pi Z}{h_{0}} \chi_{r}^{(\mu)}(r) \\
\cos \frac{m \pi Z}{h_{0}} \chi_{\theta}^{(\mu)}(r) \\
\mathrm{i} \sin \frac{m \pi Z}{h_{0}} \chi_{Z}^{(\mu)}(r) \\
\cos \frac{m \pi Z}{h_{0}} \Theta^{(\mu)}(r)
\end{array}\right],
$$

where the real functions $\chi_{r}^{(\mu)}, \chi_{\theta}^{(\mu)}, \chi_{Z}^{(\mu)}$ and $\Theta^{(\mu)}$ define the radial structure of the mode and are given in Appendix $\mathrm{A}$ in terms of the Bessel function $J_{n}\left(k^{(\mu)} r\right)$ and its derivative, while the quantity $k^{(\mu)}$ is a transverse wavenumber which depends on which of the infinity of modes of the family $n, m$ is considered. We will occasionally use the notation $n_{\mu}$ and $m_{\mu}$ to denote the indices of mode $\mu$.

Modes with $m=0$ have $\omega^{(\mu)}=0$, i.e. they do not oscillate in time, representing steady two-dimensional motion, independent of $Z$ and with $u_{Z}=0$. They are often referred to as geostrophic because there is equilibrium between the Coriolis and pressure gradient terms in (3.3) when $\omega^{(\mu)}=0$. Modes of the particular family $n=m=0$ are real and axisymmetric as well as geostrophic and play an important role in the nonlinear theory of Part 2. Their only non-zero velocity component is $u_{\theta}(r)$, corresponding to steady differential rotation of cylinders of fluid of constant $r$ about the $Z$-axis (see figure $2 a$ ).

The infinity of different geostrophic families (different values of $n$ ), each of which consists of an infinity of modes, means that the eigenfrequency $\omega=0$ is hugely degenerate (any two-dimensional incompressible velocity field with $u_{r}=0$ on $r=1$ yields a geostrophic flow). As a result, there is considerable freedom in the choice of geostrophic modes, a freedom which is exploited by the particular choice made in 
Appendix A. The $m=0$ modes defined there satisfy the no-slip condition $u_{\theta}^{(\mu)}=0$ on $r=1$. Although, because it turns out that geostrophic modes do not lead to linear instability, this property is unimportant for the purposes of the present paper, it is significant in the weakly nonlinear analysis of Part 2.

Non-geostrophic modes are oscillatory (i.e. $\omega^{(\mu)} \neq 0$ ) and any given $m \neq 0$ modal family can be subdivided into negative and positive values of $\omega^{(\mu)}$. Modes with $n=0$ are axisymmetric and can be visualized as one or more oscillatory toroidal vortices encircling the $Z$-axis (see figure $2 b, c$ ). A non-axisymmetric mode, whether geostrophic or not, has $\exp \left(\mathrm{i}\left(n \theta-\omega^{(\mu)} t\right)\right)$ dependence on $\theta$ and $t$, implying steady flow in a frame of reference rotating at velocity $\omega^{(\mu)} / n$. That is, the mode structure may be thought of as rotating about the cylinder axis, providing an interpretation of the modal oscillations as arising from the passage of the non-axisymmetric sinusoidal azimuthal structure $\mathrm{e}^{\mathrm{i} n \theta}$, past a fixed point of our chosen reference frame owing to modal rotation at angular velocity $\omega^{(\mu)} / n$.

To any mode $\mu$, there is a complex conjugate mode, denoted $\mu^{*}$. Conjugation changes the signs of $n$ and $\omega^{(\mu)}$, leaves $m$ and $k^{(\mu)}$ unchanged, and conjugates the eigenfunctions $\boldsymbol{u}^{(\mu)}$ and $\lambda^{(\mu)}$. The particular family $n=m=0$ are real modes which are their own conjugates, while $\mu$ and $\mu *$ are otherwise distinct, contributing conjugate terms to the modal expansion of a real velocity field. Although both the mode and its conjugate are mathematically necessary in such an expansion, they can be thought of as representing the same physical entity. Negative values of $n$ are the conjugates of positive ones, whereas the conjugate of an axisymmetric mode is also axisymmetric and of the same family as the original one, but with the opposite sign of frequency if $m \neq 0$. Thus, a modal family $n, m$ with negative $n$ is mathematically distinct, but physically equivalent to the family $-n, m$, while, among axisymmetric modes with $m \neq 0$, those of negative frequency are the conjugates of positive frequency ones, forming pairs of physically equivalent axisymmetric modes differing by the sign of $\omega^{(\mu)}$.

We will sometimes refer to modes having large values of $n, m$ or $k^{(\mu)}$ as highorder. Using the asymptotic expansion of the Bessel function $J_{n}$ for large argument and order, it can be shown that (in those parts of the cylinder where they are not exponentially evanescent) high-order modes consist of a combination of four locally plane inertial waves of wavenumber

$$
K^{(\mu)}=\left(k^{(\mu)^{2}}+\left(\frac{m \pi}{h_{0}}\right)^{2}\right)^{1 / 2},
$$

which is the norm of a local wavevector $\boldsymbol{K}$ having axial component $\pm m \pi / h_{0}$ and a transverse part of norm $k^{(\mu)}$. The modal frequency is related to the direction of the local wavevector by the usual dispersion relation, $\omega^{(\mu)}= \pm m \pi / K^{(\mu)} h_{0}$, for plane inertial waves. The modal wavenumber $K^{(\mu)}$ provides a quantitative measure of the order of mode $\mu$. Low-order modes have wavelengths comparable with the cylinder dimensions and $K^{(\mu)}$ of order one, but as $K^{(\mu)}$ increases, the modal wavelength, $2 \pi / K^{(\mu)}$, decreases and high-order modes consist of short waves and have large $K^{(\mu)}$. The infinite density of modal frequencies in $\left|\omega^{(\mu)}\right|<1$ remarked on earlier, reflects modes of shorter and shorter wavelengths covering the same range of frequency.

\subsection{Modal projection}

The inertial-mode eigenfunctions form an orthogonal set with respect to the usual complex inner product (Greenspan 1969, § 2.7), i.e.

$$
\int \boldsymbol{u}^{(\mu)^{*}} \cdot \boldsymbol{u}^{(\nu)} \mathrm{d}^{3} \boldsymbol{X}=\delta_{\mu \nu}
$$


where the asterisk denotes complex conjugation and the value 1 for $\mu=v$ is a consequence of the normalization adopted in Appendix A (hereinafter $v$ is a modal index, not to be confused with the kinematic viscosity, which does not appear in the remainder of these papers). A general divergence-free vector field whose normal component is zero at the boundaries of the cylinder can be written as a linear combination of the inertial-mode eigenfunctions. In particular, the velocity perturbation may be expanded as

$$
\boldsymbol{u}=\sum_{\mu} B_{\mu}(t) \boldsymbol{u}^{(\mu)}(\boldsymbol{X}),
$$

where $B_{\mu}$ are modal amplitudes which, using (3.6), are given by

$$
B_{\mu}=\int \boldsymbol{u}^{(\mu)^{*}} \cdot \boldsymbol{u} \mathrm{d}^{3} \boldsymbol{X} .
$$

Since $\boldsymbol{u}$ is real and $\boldsymbol{u}^{\left(\mu^{*}\right)}=\boldsymbol{u}^{(\mu)^{*}}, B_{\mu}^{*}=B_{\mu^{*}}$, i.e. the amplitudes of a mode and its conjugate are complex conjugates, a necessary and sufficient condition for the $B_{\mu}$ to represent a real perturbation and which reflects the physical equivalence of conjugate modes discussed earlier.

An evolution equation for $B_{\mu}$ can be derived by scalar multiplication of (2.18) by $\boldsymbol{u}^{(\mu)^{*}}$ followed by integration over the cylinder volume. All spatial derivatives are transferred onto $\boldsymbol{u}^{(\mu)^{*}}$ using the divergence theorem, (2.10), (2.12), (2.17), (3.2), (3.3) and (3.8) and the boundary conditions on $\boldsymbol{u}$ and $\boldsymbol{u}^{(\mu)}$. Finally, $\boldsymbol{V}$ is written as the sum of $\boldsymbol{U}$ and $\boldsymbol{u}$, leading to

$$
\begin{aligned}
& \frac{\mathrm{d} B_{\mu}}{\mathrm{d} t}+\mathrm{i} \omega^{(\mu)} B_{\mu}=\underbrace{\frac{\mathrm{d}}{\mathrm{d} t}\left\{\left(1-\left(\frac{h}{h_{0}}\right)^{2}\right) \int u_{Z}^{(\mu)^{*}} u_{Z} \mathrm{~d}^{3} \boldsymbol{X}\right\}}_{\text {Piston motion }} \\
& +R e^{-1}\{\underbrace{\int_{r=1}\left(\boldsymbol{u}_{\perp}^{(\mu)^{*}}+\left(\frac{h}{h_{0}}\right)^{2} \boldsymbol{e}_{Z} u_{Z}^{(\mu)^{*}}\right) \cdot(\boldsymbol{n} \cdot \nabla \boldsymbol{u}) \mathrm{d}^{2} \boldsymbol{X}}_{\text {Side-wall viscous term }} \\
& +\underbrace{\int_{Z=0, h_{0}}\left(\left(\frac{h_{0}}{h}\right)^{2} \boldsymbol{u}_{\perp}^{(\mu)^{*}}+\boldsymbol{e}_{Z} u_{Z}^{(\mu)^{*}}\right) \cdot(\boldsymbol{n} \cdot \nabla \boldsymbol{u}) \mathrm{d}^{2} \boldsymbol{X}}_{\text {End-wall viscous term }} \\
& +\underbrace{\int \boldsymbol{u} \cdot \mathscr{D}\left(\boldsymbol{u}_{\perp}^{(\mu)^{*}}+\left(\frac{h}{h_{0}}\right)^{2} \boldsymbol{e}_{Z} u_{Z}^{(\mu)^{*}}\right) \mathrm{d}^{3} \boldsymbol{X}}_{\text {Volumetric viscous term }}\} \\
& +\int[\underbrace{\boldsymbol{u} \cdot(\boldsymbol{u} \cdot \nabla)}_{\text {Nonlinearity }}+\underbrace{\boldsymbol{U} \cdot(\boldsymbol{u} \cdot \nabla)+\boldsymbol{u} \cdot(\boldsymbol{U} \cdot \nabla)}_{\begin{array}{c}
\text { Effects of viscosity } \\
\text { on the basic flow }
\end{array}}] \\
& \times\left(\boldsymbol{u}_{\perp}^{(\mu)^{*}}+\left(\frac{h}{h_{0}}\right)^{2} \boldsymbol{e}_{Z} u_{Z}^{(\mu)^{*}}\right) \mathrm{d}^{3} \boldsymbol{X},
\end{aligned}
$$


where $\boldsymbol{n}$ is an outward unit normal at the cylinder boundary. As indicated by the annotation, the right-hand side of (3.9) consists of three groups of terms representing: (i) piston motion; (ii) viscous effects and (iii) a combination of nonlinearity (the $\boldsymbol{u} \cdot(\boldsymbol{u} \cdot \nabla)$ term) and linear terms involving the departure, $\boldsymbol{U}$, between the viscous and inviscid basic flows. The viscous terms can be further subdivided into surface and volumetric contributions.

The piston motion term in (3.9) can be immediately expressed in terms of the $B_{\mu}$ using (3.7):

$$
\int u_{Z}^{(\mu)^{*}} u_{Z} \mathrm{~d}^{3} \boldsymbol{X}=\sum_{\nu} C_{\mu \nu} B_{\nu},
$$

where $C_{\mu \nu}=\int u_{Z}^{(\mu)^{\circ}} u_{Z}^{(\nu)} \mathrm{d}^{3} \boldsymbol{X}$ is a real symmetric matrix representing modal coupling by piston motion and whose elements satisfy $\left|C_{\mu \nu}\right|<1$. Since, as noted earlier, instability is due to piston motion, the coupling coefficients $C_{\mu \nu}$ are crucial to the present study and details are given in Appendix A. It is found that: (i) piston motion only couples modes $\mu, v$ of the same family; (ii) there is no coupling of geostrophic modes $(m=0)$; (iii) an axisymmetric mode $(n=0)$ only couples to itself and its conjugate; and (iv) when $n \neq 0$ and $m>0$, all modes in the family are coupled. As we shall see, these properties of $C_{\mu \nu}$ have important consequences for stability.

\section{Asymptotics for small sinusoidal piston motion}

From now on, we consider

$$
h=h_{0}\left(1+\varepsilon \cos \omega_{0} t\right),
$$

where $\omega_{0}>0$ is the piston frequency and the piston amplitude, $\varepsilon>0$, is a small parameter on which subsequent asymptotics are based. Since piston motion is the source of instability, small $\varepsilon$ implies low growth rates. Viscosity has a damping effect on the instabilities and must therefore also be small so as not to kill them altogether. As we shall see, the appropriate Reynolds-number scaling, making viscous effects comparable with those of piston motion, is $R e=O\left(\varepsilon^{-2}\right)$ and this is supposed in what follows. As usual in rotating flows, the boundary-layer thickness scales as $R e^{-1 / 2}$ at large Reynolds number (Greenspan 1969, §2.3, recalling that the Ekman number used there is the inverse of the rotational Reynolds number used in this paper). The assumed scaling thus implies a boundary-layer thickness $O(\varepsilon)$.

The modal amplitudes of the perturbation are expanded as

$$
B_{\mu}=\delta\left(B_{\mu}^{[1]}+\varepsilon B_{\mu}^{[2]}+\ldots\right),
$$

where $\delta$ is a parameter determining the perturbation amplitude which is sufficiently small that nonlinearity can be neglected and the coefficients $B_{\mu}^{[i]}$ are functions of the fast and slow times $t$ and $T=\varepsilon t$. All terms on the right-hand side of (3.9) are of smaller order than $\delta$ and so

$$
\frac{\partial B_{\mu}^{[1]}}{\partial t}+\mathrm{i} \omega^{(\mu)} B_{\mu}^{[1]}=0,
$$

at leading order. The solution of (4.3) is

$$
B_{\mu}^{[1]}=A_{\mu}(T) \exp \left(-\mathrm{i} \omega^{(\mu)} t\right),
$$

so each mode oscillates with its natural frequency at leading order and on the fast time scale. Observe that, since $\omega^{\left(\mu^{*}\right)}=-\omega^{(\mu)}$, the conjugacy relation $A_{\mu^{*}}=A_{\mu}^{*}$ follows 
from that of the $B_{\mu}$. To derive equations for the slowly varying $A_{\mu}(T)$, we go to the next order. To this effect, we examine each term on the right-hand side of (3.9).

\subsection{Derivation of the mode-pair amplitude equations}

The piston-motion term is evaluated using (3.10), (4.1), (4.2) and (4.4) as

$$
\begin{aligned}
\frac{\mathrm{d}}{\mathrm{d} t}\left\{\left(1-\left(\frac{h}{h_{0}}\right)^{2}\right) \int u_{Z}^{(\mu)^{*}} u_{Z} \mathrm{~d}^{3} X\right\} \sim & \mathrm{i} \delta \varepsilon \sum_{\nu} C_{\mu \nu} A_{\nu}\left\{\left(\omega^{(\nu)}-\omega_{0}\right) \exp \left(-\mathrm{i}\left(\omega^{(\nu)}-\omega_{0}\right) t\right)\right. \\
& \left.+\left(\omega^{(\nu)}+\omega_{0}\right) \exp \left(-\mathrm{i}\left(\omega^{(\nu)}+\omega_{0}\right) t\right)\right\}
\end{aligned}
$$

correct to order $\delta \varepsilon$, where we have written $\cos \omega_{0} t$ as a sum of exponentials. The viscous terms are treated as follows. The Reynolds number is replaced by its time average $\overline{R e}$, becoming a constant parameter from here on, while the quantity $h / h_{0}$ is replaced by 1 in (2.12) and all viscous terms of (3.9). Boundary-layer analysis (Appendix B) is then used to evaluate the surface integral terms as

$$
\overline{R e}^{-1} \int \boldsymbol{u}^{(\mu)^{*}} \cdot(\boldsymbol{n} \cdot \nabla \boldsymbol{u}) \mathrm{d}^{2} \boldsymbol{X} \sim-\delta \overline{R e}^{-1 / 2} \sum_{\nu} D_{\mu \nu} A_{\nu} \exp \left(-\mathrm{i} \omega^{(\nu)} t\right),
$$

again correct to order $\delta \varepsilon$, where $D_{\mu \nu}$ is a complex coupling matrix representing surface viscous effects, some details of which are given in Appendix B. Only modes with the same angular order $n$ are coupled by $D_{\mu \nu}$. Finally, the volumetric viscous term is evaluated using (A 8), with the help of (2.17), (3.8), (4.2) and (4.4), the divergence theorem and the boundary conditions on $\boldsymbol{u}$, as

$$
\overline{R e}^{-1} \int \boldsymbol{u} \cdot \nabla^{2} \boldsymbol{u}^{(\mu)^{*}} \mathrm{~d}^{3} X \sim-\delta \overline{\operatorname{Re}}^{-1} K^{(\mu)^{2}} A_{\mu} \exp \left(-\mathrm{i} \omega^{(\mu)} t\right) .
$$

Although (4.7) is formally of order $\delta \varepsilon^{2}$ (one order smaller than the other terms), as discussed in $\S 1$, we include it at the present order to allow a uniform treatment of the higher-order modes. That is, (4.7) becomes $O(\delta \varepsilon)$, if $K^{(\mu)}$ is large $O\left(\varepsilon^{-1 / 2}\right)$, and we treat it as if it were the same order as (4.5) and (4.6) in what follows. Note that, like the modal eigenvalue problem, the matrices $C_{\mu \nu}$ and $D_{\mu \nu}$ depend only on $h_{0}$.

The nonlinear term in (3.9) is neglected because the perturbation amplitude $\delta$ is assumed sufficiently small and the terms involving $\boldsymbol{U}$ for the following reasons. As discussed earlier, $\boldsymbol{U}$ is the difference between the basic flow with and without viscosity and consists of a side-wall boundary layer, corner flows and secondary flows in the cylinder interior owing to Ekman pumping. $\boldsymbol{U}$ is $O(\varepsilon)$ in the boundary-layer and corner regions, which occupy a volume $O(\varepsilon)$ of the cylinder and hence contribute $O\left(\delta \varepsilon^{2}\right)$ to the $\boldsymbol{U}$-terms in (3.9). The volume flux deficit in the basic-flow boundary layer is $O\left(\varepsilon^{2}\right)$ (a velocity deficit of $O(\varepsilon)$ multiplying a layer thickness of the same order). Ekman pumping thus drives the secondary flow with a forcing $O\left(\varepsilon^{2}\right)$, suggesting a contribution $O\left(\delta \varepsilon^{2}\right)$ to (3.9) from the cylinder interior. However, this estimate can be too conservative. If the piston frequency $\omega_{0}$ happens to be close to one of the low-order axisymmetric modal frequencies, the resonant response of the mode is larger than the $O\left(\varepsilon^{2}\right)$ forcing would suggest, rising to $O(\varepsilon)$ if $\omega_{0}$ matches a low-order modal frequency to within the modal damping bandwidth $O(\varepsilon)$. This would yield a contribution to (3.9) of $O(\delta \varepsilon)$, comparable with (4.5), (4.6) and too large to be neglected. However, at small $\varepsilon$, fine tuning of the cylinder geometry is required to make a low-order axisymmetric mode satisfy the direct resonance condition to $O(\varepsilon)$ and we suppose this is not the case. With this proviso, the contribution of the $\boldsymbol{U}$-terms to $(3.9)$ is $o(\delta \varepsilon)$, negligible compared to (4.5) and (4.6). 
Collecting together the above results, the $O(\delta \varepsilon)$ problem arising from (3.9) can be written as

$$
\frac{\partial B_{\mu}^{[2]}}{\partial t}+\mathrm{i} \omega^{(\mu)} B_{\mu}^{[2]}=\Psi_{\mu}-\frac{\mathrm{d} A_{\mu}}{\mathrm{d} T} \exp \left(-\mathrm{i} \omega^{(\mu)} t\right),
$$

where $\Psi_{\mu}$ is the sum of the right-hand sides of (4.5)-(4.7), divided by $\delta \varepsilon$. As usual, the amplitude equations follow from imposing non-secularity conditions. The right-hand side of (4.8) consists of a sum of oscillatory exponentials and secular terms in $B_{\mu}^{[2]}$ arise from those exponentials which satisfy a fast-time resonance condition, i.e. their frequency coincides with $\omega^{(\mu)}$ to $O(\varepsilon)$. For instance, the second term on the right of (4.8) is always resonant, as are (4.7) and the $v=\mu$ term of (4.6). If these are the only resonant terms, the non-secularity condition is

$$
\frac{\mathrm{d} A_{\mu}}{\mathrm{d} T}=-\varepsilon^{-1} \overline{R e}^{-1 / 2} d_{\mu} A_{\mu},
$$

where

$$
d_{\mu}=D_{\mu \mu}+\overline{R e}^{-1 / 2} K^{(\mu)^{2}}
$$

is the complex viscous damping factor, $d_{\mu}=d_{\mu}^{r}+\mathrm{i} d_{\mu}^{i}$, of mode $\mu$. The real part of $D_{\mu \mu}=D_{\mu \mu}^{r}+\mathrm{i} D_{\mu \mu}^{i}$ is positive and thus both boundary-layer and volumetric contributions to $d_{\mu}^{r}$ are positive. According to (4.9) with positive $d_{\mu}^{r}$, the mode decays and oscillates with a frequency which undergoes a small viscous correction $\overline{R e}^{-1 / 2} d_{\mu}^{i}$ to its inviscid value $\omega^{(\mu)}$. This almost corresponds with the classical theory of inertial-mode damping by viscosity (Greenspan 1969, §2.9), which yields the boundary-layer modal damping factor $\overline{R e}^{-1 / 2} D_{\mu \mu}^{r}$ and frequency correction $\overline{R e}^{-1 / 2} D_{\mu \mu}^{i}$, but with the significant addition of the volumetric term containing $K^{(\mu)}$ in (4.10).

For there to be instability, piston-motion must play a role in the amplitude equations. Thus, (4.5) should contribute secular forcing to (4.8) for at least one mode $\mu$. This implies the existence of a pair of modes, $\mu_{+}$and $\mu_{-}$say, which satisfy the parametric resonance condition that the difference of their modal frequencies matches $\omega_{0}$ to $O(\varepsilon)$ and have a non-zero coupling coefficient $C=C_{\mu_{+} \mu_{-}}=C_{\mu_{-} \mu_{+}}$. Interchanging the two modes if necessary, the resonance condition can be written

$$
\omega_{0}=\omega_{+}-\omega_{-}+\varepsilon \Delta,
$$

where $\omega_{+}=\omega^{\left(\mu_{+}\right)}, \omega_{-}=\omega^{\left(\mu_{-}\right)}$and $\Delta$ is an $O(1)$ detuning parameter expressing the departure from exact resonance. Focusing attention on (4.8) with $\mu=\mu_{+}$and $\mu=\mu_{-}$ and supposing there are no resonant terms other than those identified above (a question we will return to in the discussion below), the non-secularity conditions for the two modes yield

$$
\begin{aligned}
& \frac{\mathrm{d} A_{+}}{\mathrm{d} T}=\mathrm{i} \omega_{+} C \mathrm{e}^{-\mathrm{i} \Delta T} A_{-}-\hat{d}_{+} A_{+}, \\
& \frac{\mathrm{d} A_{-}}{\mathrm{d} T}=\mathrm{i} \omega_{-} C \mathrm{e}^{\mathrm{i} \Delta T} A_{+}-\hat{d}_{-} A_{-},
\end{aligned}
$$

where $\hat{d}_{ \pm}=\varepsilon^{-1} \overline{R e}-1 / 2 d_{ \pm}, d_{ \pm}=d_{\mu_{ \pm}}, A_{ \pm}=A_{\mu_{ \pm}}$and we have used (4.11) in the $\omega^{(v)} \pm \omega_{0}$ multiplicative factors of the resonant terms of (4.5). Given the properties of $C_{\mu \nu}$ noted at the end of $\S 3$, non-zero $C$ implies that $\mu_{+}$and $\mu_{-}$must not only satisfy (4.11), but also (i) belong to the same $m \neq 0$ family (same $n$ and $m$ ), and (ii) be conjugates $\left(\mu_{-}=\mu_{+}^{*}\right)$ if they are axisymmetric. These are important constraints on possible mode pairs. The mode-pair equations (4.12) and (4.13) form the basis for the stability analysis in the next section. 


\subsection{Discussion}

\subsubsection{Out-of-pair coupling}

Resonant interactions with other modes might arise from either (4.5) or (4.6). Significant coupling to a third mode $v$ via (4.5) requires (i) that parametric resonance between $v$ and one of the modes of the pair, i.e. either $\left|\omega_{\nu}-\omega_{+}\right|$or $\left|\omega_{\nu}-\omega_{-}\right|$coincide with $\omega_{0}$ to $O(\varepsilon)$, and (ii) that the corresponding coupling coefficient, $C_{\mu_{+} v}$ or $C_{\mu_{-} v}$, not be small or zero, for otherwise the resonant term in (4.5) is $o(\delta \varepsilon)$ and need not be included at the present order. Such coupling is impossible for axisymmetric mode pairs, because $C_{\mu_{+\nu}}=C_{\mu_{-\nu}}=0$ for all modes $v$ not already in the pair. On the other hand, if $n \neq 0$, all $v$ which are members of the same modal family as the pair are possible candidates. However, the parametric resonance condition means that $\omega^{(v)}$ must fall within one of four small frequency ranges of width $O(\varepsilon)$ and this becomes increasingly hard for modes in the given family to satisfy as $\varepsilon \rightarrow 0$. Unless the cylinder aspect ratio has been specially chosen to tune one of the $\omega^{(v)}$ and provided $\varepsilon$ is sufficiently small, there should be no third-mode coupling by piston motion.

Significant coupling of a third mode via (4.6) requires (i) coincidence to $O(\varepsilon)$ of $\omega^{(v)}$ with either $\omega_{+}$or $\omega_{-}$, and (ii) that the corresponding coupling coefficient, $D_{\mu_{+} \nu}$ or $D_{\mu_{-} v}$, not be small or zero. The first condition requires $\omega^{(v)}$ to lie in one of two small frequency ranges of width $O(\varepsilon)$. Non-zero $D_{\mu_{+}}$implies that $v$ has the same angular order $n$ as the mode pair, but this leaves open the infinity of modal families obtained by varying $m$. Unlike those of a single family, possible modal frequencies are dense in the interval $\left|\omega^{(v)}\right|<1$, leading to an infinite number of modes satisfying the resonance condition. However, infinite modal density arises from modes of higher and higher orders and it can be shown that the coupling coefficients $D_{\mu_{+} \nu}$ and $D_{\mu_{-} \nu}$ tend to zero for high-order $v$, hence significant resonances should not occur if $\varepsilon$ is sufficiently small unless the cylinder geometry has been specially chosen. It is worth remarking that the classical theory of inertial-mode damping also implicitly supposes lack of significant resonant coupling of inviscid modes by viscosity, which should lend confidence to our neglect of such coupling here.

\subsubsection{Volumetric damping}

As noted earlier, the volumetric term in (4.10) does not appear in the classical theory of viscous damping because that theory is based on large-Reynolds-number asymptotics and the volumetric term is, after all, multiplied by $\overline{R e}^{-1 / 2}$. It is maintained here to allow a uniform treatment of higher-order modes and it is perhaps worth assessing the importance of the volumetric contribution to viscous damping.

Kerswell \& Barenghi (1994) undertook a numerical study of the linearized incompressible Navier-Stokes equations in a rotating cylinder without piston motion. They compared their results with the modal damping factors obtained from classical theory and observed discrepancies at lower (but still high) Reynolds numbers. Le Noble (1995) carried out a similar numerical study and found that adding volumetric damping greatly improved the agreement. Kerswell (1999, figure 10) came to the same conclusion. To fix ideas, when $\overline{R e}=10^{4}$ and $h_{0}=2$, the volumetric term contributes $14 \%, 34 \%$ and $52 \%$ to the total damping of the first three modes of the family $n=0$, $m=1$. Thus, it is far from negligible for the third mode, which is still of relatively low order.

On the subject of modal damping, there is some confusion in the literature concerning the correct expression for the boundary-layer damping factors $\left(D_{\mu \mu}\right)$ of a cylinder, with different authors reporting differing results. Kerswell \& Barenghi 
(1994) compared two such expressions with their numerical results and concluded that their equation (2.12) (due to Kudlick (1966) and in agreement with our results) was in better agreement with their simulations in the limit of large Reynolds number. The problem appears to have arisen from insufficient care in taking the limit of the classical results for a general container (Greenspan 1969, (2.9.12), (2.9.13)) as the singular case of the cylinder is approached. Note that the present approach, based on modal projection, avoids such difficulties.

\section{Instability criteria and results}

\subsection{Instability criteria}

Equations (4.12), (4.13) have exponential solutions of the form

$$
A_{+}=a_{+} \mathrm{e}^{(s-\mathrm{i} \Delta / 2) T}, \quad A_{-}=a_{-} \mathrm{e}^{(s+\mathrm{i} \Delta / 2) T},
$$

where the constants $a_{ \pm}$satisfy a pair of linear equations, the condition for a non-trivial solution of which yields the quadratic equation

$$
\hat{s}^{2}+\left(\hat{d}_{+}^{r}+\hat{d}_{-}^{r}\right) \hat{s}+\hat{d}_{+}^{r} \hat{d}_{-}^{r}+\omega_{+} \omega_{-} C^{2}+\frac{1}{4} \hat{\Delta}^{2}+\frac{1}{2} \mathrm{i} \hat{\Delta}\left(\hat{d}_{+}^{r}-\hat{d}_{-}^{r}\right)=0,
$$

for $\hat{s}=s+\frac{1}{2} i\left(\hat{d}_{+}^{i}+\hat{d}_{-}^{i}\right)$, where $\hat{\Delta}=\varepsilon^{-1}\left(\omega_{0}-\omega_{c}\right)$ is a modified detuning parameter expressing the scaled departure of the piston frequency from the viscous parametric resonance condition $\omega_{0}=\omega_{c}$ and

$$
\omega_{c}=\omega_{+}+\overline{R e}^{-1 / 2} d_{+}^{i}-\left(\omega_{-}+\overline{R e}^{-1 / 2} d_{-}^{i}\right)
$$

is the difference between the mode frequencies of the pair, corrected for viscous effects.

Instability implies a growing solution, corresponding to a root of (5.2) with a positive real part. It can be shown that this requires

$$
\hat{\Delta}^{2}<-\left(\hat{d}_{+}^{r}+\hat{d}_{-}^{r}\right)^{2}\left\{1+\frac{\omega_{+} \omega_{-} C^{2}}{\hat{d}_{+}^{r} \hat{d}_{-}^{r}}\right\} .
$$

If $C^{2} \omega_{+} \omega_{-} \geqslant 0$, this condition cannot be satisfied and instability never occurs for the given mode pair, whereas when $C^{2} \omega_{+} \omega_{-}<0$, the pair grows provided

$$
\varepsilon>\varepsilon_{c}\left[1+\overline{\operatorname{Re}}\left(\frac{\omega_{0}-\omega_{c}}{d_{+}^{r}+d_{-}^{r}}\right)^{2}\right]^{1 / 2},
$$

where we have re-expressed $\hat{\Delta}$ and $\hat{d}_{ \pm}^{r}$ in (5.4) using their definitions and introduced

$$
\varepsilon_{c}=\left[-\frac{d_{+}^{r} d_{-}^{r}}{\overline{R e} \omega_{+} \omega_{-} C^{2}}\right]^{1 / 2} .
$$

Thus, instability occurs if $C^{2} \omega_{+} \omega_{-}<0$ and (5.5) holds. $C^{2} \omega_{+} \omega_{-}<0$ selects mode pairs with $C \neq 0$ and oppositely signed frequencies $\left(\omega_{+}>0\right.$ and $\left.\omega_{-}<0\right)$, while (5.5) yields a neutral hyperbola in the $\left(\omega_{0}, \varepsilon\right)$-plane illustrated in figure 3 . The lowest point of the hyperbola occurs at $\omega_{0}=\omega_{c}, \varepsilon=\varepsilon_{c}$ and yields a critical piston amplitude $\varepsilon_{c}$ below which instability cannot occur no matter what the piston frequency. When $\varepsilon>\varepsilon_{c}$, there is a band of piston frequencies centred on $\omega_{0}=\omega_{c}$ for which the mode pair has unstable exponential growth. Both $\varepsilon_{c}$ and the width of the unstable band are small, $O\left(\overline{R e}^{-1 / 2}\right)$. 


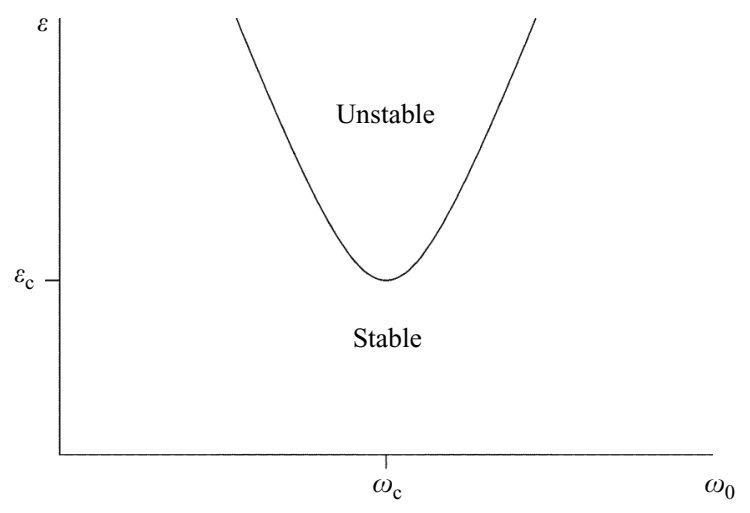

FIGURE 3. Sketch of the neutral curve (hyperbola) of a single mode pair in the $\omega_{0}$ (piston frequency), $\varepsilon$ (piston amplitude) plane for given values of $\overline{R e}$ and $h_{0}$.

A summary is given below.

(i) Geostrophic modes do not lead to coupled mode pairs, a prerequisite for instability, and for this reason are not considered further in this paper. However, as we will see in Part 2, the particular family $n=m=0$ turns out to be important in the weakly nonlinear theory.

(ii) Conjugate axisymmetric modes form coupled mode pairs and, what is more, have oppositely signed frequencies $\omega_{-}=-\omega_{+}$. In consequence, they are unstable within the neutral hyperbola (5.5), where, since $d_{-}=d_{+}^{*}$ and $C=-\frac{1}{2}\left(1-\omega_{+}^{2}\right)$,

$$
\varepsilon_{c}=\frac{2 \overline{R e}^{-1 / 2} d_{+}^{r}}{\omega_{+}\left(1-\omega_{+}^{2}\right)}, \quad \omega_{c}=2\left(\omega_{+}+\overline{R e}^{-1 / 2} d_{+}^{i}\right),
$$

give the base of the neutral curve. The result of instability is a growing mode pair, physically equivalent to a single axisymmetric mode, $\mu_{+}$. As discussed earlier, the associated velocity perturbation consists of one or more oscillating toroidal vortices encircling the $Z$-axis (recall figures $2 b$ and $2 c$ ). Since $\left|\omega_{0}-\omega_{c}\right|=O(\varepsilon)$ for instability, the oscillation frequency is close to half the piston frequency according to (5.7). It should be borne in mind that, in addition to the $u_{r}$ and $u_{Z}$ components illustrated by figures $2(b)$ and $2(c)$, the mode involves a $u_{\theta}$ component, whose oscillations are $\pi / 2$ out of phase with those of $u_{r}, u_{Z}$ owing to the factors of $i$ in (3.4).

(iii) In the non-axisymmetric case $n \neq 0$, the modes are no longer conjugates and represent distinct physical entities, which changes the nature of the flow resulting from instability. The modes must belong to the same family and their frequencies must have opposite signs, conditions which place restrictions on possible mode pairs, but which are less severe than the conjugacy requirement of axisymmetric modes. Instability occurs if (5.5) holds, implying a growing mode pair whose frequency difference is close to the piston frequency. Since modes in the pair are not conjugates, a physically meaningful (i.e. real) solution also involves the conjugate mode pair. Thus, four modes are mathematically necessary to express a real perturbation, of which just two are physically distinct. It is more difficult to visualize the velocity perturbation associated with non-axisymmetric instability, partly because the modes themselves are harder to visualize, but also because it involves a superposition of two modes of different frequencies and radial structures, whose velocity perturbations interfere in a complicated time-dependent manner. 
In the limit $\overline{R e} \rightarrow \infty,(4.10),(5.5)$ and (5.6) yield the limiting neutral curve

$$
\varepsilon=\left[-\frac{\Xi}{\omega_{+} \omega_{-} C^{2}(\Xi+1)^{2}}\right]^{1 / 2}\left|\omega_{0}-\omega_{c}\right|,
$$

where $\Xi=D_{\mu_{+} \mu_{+}}^{r} / D_{\mu_{-} \mu_{-}}^{r}$. Thus, viscosity still appears in the problem in the largeReynolds-number limit via the ratio, $\Xi$, of boundary-layer modal damping factors. The neutral curve (5.8) consists of two straight lines originating at $\varepsilon=0, \omega_{0}=\omega_{c}$ $\left(=\omega_{+}-\omega_{-}\right.$in the infinite $\overline{R e}$ limit considered here $)$ in the $\left(\omega_{0}, \varepsilon\right)$-plane and the unstable region is a vertical straight-sided wedge. There is no minimum piston amplitude for instability since $\varepsilon_{c} \rightarrow 0$ as $\overline{R e} \rightarrow \infty$.

Equation (5.8) does not generally coincide with the neutral curve obtained from inviscid analysis. The inviscid equivalent of (5.2) is obtained by setting $\hat{d}_{ \pm}^{r}=0$. One of the roots of the resulting equation has a positive real part inside the inviscid neutral wedge bounded by

$$
\varepsilon=\left(-4 \omega_{+} \omega_{-} C^{2}\right)^{-1 / 2}\left|\omega_{0}-\omega_{c}\right|
$$

which has the same generic form as (5.8), with the same $\omega_{c}=\omega_{+}-\omega_{-}$, but the slopes of the straight lines differ unless $\Xi=1$. Whereas $\Xi=1$ for axisymmetric mode pairs (because they consist of conjugate modes) this is not generally the case for nonaxisymmetric ones. When $\Xi \neq 1$, the unstable region given by (5.8) is larger than the inviscid one, i.e. the addition of non-zero viscosity can destabilize non-axisymmetric mode pairs. Outside (5.9), the growth/decay rates of viscous theory tend to zero in the limit $\overline{R e} \rightarrow \infty$, in which sense the inviscid case is approached, but instability persists inside the region bounded by (5.8). Although, as we have just seen, the inclusion of viscosity can destabilize a non-axisymmetric mode pair in the limit of infinite Reynolds number, at finite $\overline{R e}$ it can be either stabilizing or destabilizing. Both the width and centre of the unstable band of piston frequencies are altered by viscosity and a particular operating point in the $\left(\omega_{0}, \varepsilon\right)$-plane may find itself taken from inside (5.9) to lie outside the neutral curve when viscosity is introduced, or vice versa. What is more, within the viscous theory, mode pairs (even axisymmetric ones) can lose stability as $\overline{R e}$ is decreased at fixed $\omega_{0}, \varepsilon$. However, viscosity is always stabilizing in the sense that it gives rise to a critical piston amplitude, zero at infinite $\overline{R e}$ and increasing with decreasing $\overline{R e}$ according to (4.10) and (5.6), below which the pair is stable at all piston frequencies. As we shall see, when the overall stability of the flow is examined, it is this stabilizing effect of viscosity, rather than the possible destabilization of particular mode pairs at particular values of $\omega_{0}$, $\varepsilon$, which is more significant.

\subsection{Detailed results}

To determine the overall stability of the flow, all mode pairs satisfying $\omega_{+} \omega_{-} C^{2}<0$ must be included. Figure 4 shows the neutral curves in the $\left(\omega_{0}, \varepsilon\right)$-plane when $\overline{R e}=10^{4}$ and $h_{0}=2$ (i.e. a cylinder of square cross-section). Qualitatively similar results are found for other values of $\overline{R e}$ and $h_{0}$. The flow is linearly stable at low enough piston amplitudes, having the potential for instability with appropriate choice of $\omega_{0}$ once $\varepsilon$ exceeds the bottom of the lowest of the hyperbolae. If $\omega_{0}, \varepsilon$ lie inside just one hyperbola, the corresponding mode pair grows, whereas, where two hyperbolae overlap there are two growing mode pairs, and so on. As $\varepsilon$ is increased, there are more and more overlapping bands of instability in $\omega_{0}$, reflecting the destabilizing effect of raising the piston amplitude. Many, but not all, of the lower hyperbolae in the figure correspond to axisymmetric modes (thicker lines). This reflects a general 


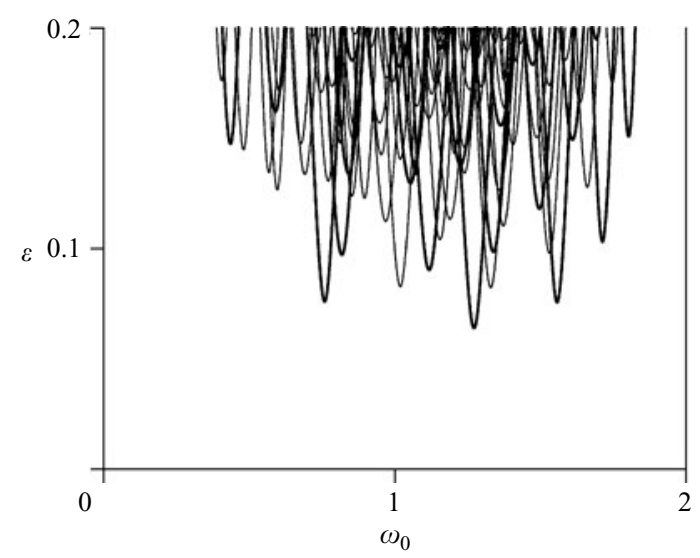

FiguRE 4. Neutral curves in the $\left(\omega_{0}, \varepsilon\right)$-plane for all modes $\left(\overline{R e}=10^{4}, h_{0}=2\right)$. The thicker curves represent axisymmetric modes.

trend to greater stability at larger $n$ which is a consequence of shorter wavelengths, hence increased volumetric damping.

It is apparent from figure 4 that instability is confined to $\omega_{0}<2$ (corresponding to dimensional piston frequencies below $4 \Omega$ ), the range spanned by taking the difference of two inviscid mode frequencies. There is a striking trend for the base, $\varepsilon_{c}$, of the hyperbolae to rise as the limiting values $\omega_{0}=0$ and $\omega_{0}=2$ are approached, indicating greater stability at such piston frequencies. Since $\omega_{+}$and $\omega_{-}$are oppositely signed for instability, values of $\omega_{0} \approx \omega_{+}-\omega_{-}$near $\omega_{0}=0$ and $\omega_{0}=2$ correspond, respectively, to mode pairs near $\omega=0$ and $\omega= \pm 1$. Stabilization near these limiting values of $\omega_{0}$ reflects weakness of piston-motion coupling of mode pairs near $\omega=0$ and $\omega= \pm 1$. Weakness of coupling near $\omega=0$ stems from the smallness of $\omega_{ \pm}$in (4.12), (4.13), which can, in turn, be traced back via the factors $\omega^{(v)} \pm \omega_{0}$ in $(4.5)$ to the time derivative on the piston-motion term in (3.9). On the other hand, modes which are close to $\omega= \pm 1$ are of high order and the locally plane waves from which they are constituted have wavevectors which are nearly in the $Z$-direction according to the inertial-wave dispersion relation, $\omega= \pm K_{Z} /|\boldsymbol{K}|$. As a result, the modal velocities, which are perpendicular to the wavevector for a plane wave, are dominantly in directions other than $Z$, making the coefficient $C=C_{\mu_{+} \mu_{-}}=\int u_{Z}^{\left(\mu_{+}\right)^{*}} u_{Z}^{(\mu-)} \mathrm{d}^{3} \boldsymbol{X}$ small (recall that modes are normalized based on $\left|\boldsymbol{u}^{(\mu)}\right|$, which includes all components of velocity). Small $C$ implies weak piston-motion coupling for modes close to $\omega= \pm 1$, leading to stabilization near $\omega_{0}=2$.

Another reason for the stabilizing trend near $\omega_{0}=0$ and $\omega_{0}=2$ is that the corresponding mode pairs are of high order, and volumetric damping, proportional to $K^{2}$, is larger for such modes. Of course, the higher the Reynolds number, the larger the value of $K^{2}$ required to make volumetric damping significant compared with boundary-layer damping in (4.10) and the less important this second mechanism becomes. Stabilization of mode pairs of sufficiently high order by volumetric damping plays a still more important role away from $\omega_{0}=0$ and $\omega_{0}=2$. It causes the hyperbolae in the $\left(\omega_{0}, \varepsilon\right)$ plane to rise as the mode order is increased, less rapidly so at higher Reynolds numbers. In consequence, the lowest hyperbolae (lowest $\varepsilon_{c}$ ) correspond to low-order mode pairs.

The effect of neglecting the volumetric contribution to modal damping is illustrated by the dashed curve in figure 5 . The entire region above this curve is densely covered 


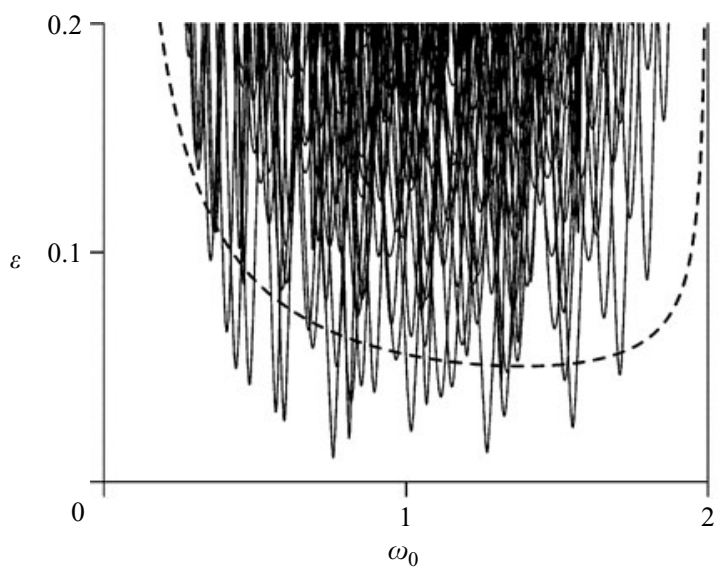

FiguRE 5. Neutral curves in the $\left(\omega_{0}, \varepsilon\right)$-plane for all modes $\left(\overline{R e}=10^{4}, h_{0}=2\right)$ when boundary-layer damping is neglected. Dropping volumetric damping instead, the region above the dashed curve (given by (5.10)) is densely filled by neutral curves.

by high-order neutral curves which were pushed off the top of figure 4 by volumetric damping. Comparing the results with and without volumetric damping, it should be clear that the inclusion of the volumetric term in the viscous damping is crucial, avoiding as it does instability to perturbations of shorter and shorter wavelengths. The lower boundary of the unstable region without volumetric damping is found to be

$$
\varepsilon=2 \overline{R e}^{-1 / 2}\left\{\omega_{0}^{-1 / 2}+\frac{\left(2-\omega_{0}\right)^{3 / 2}+\left(2+\omega_{0}\right)^{3 / 2}}{h_{0} \omega_{0}\left(4-\omega_{0}^{2}\right)^{1 / 2}}\right\},
$$

an expression which can be derived by considering the special case $n=0$. Without volumetric damping, (5.7) yields $\varepsilon_{c}=2 \overline{R e}^{-1 / 2} D_{\mu_{+} \mu_{+}}^{r} \omega_{+}^{-1}\left(1-\omega_{+}^{2}\right)^{-1}$ for the base of the neutral curves as a function of $\omega_{+}$. Evaluating $D_{\mu_{+} \mu_{+}}^{r}$ using (B 6) and replacing $\omega_{+}$ by $\omega_{0} / 2$ (exact inviscid parametric resonance) and $\varepsilon_{c}$ by $\varepsilon$ gives (5.10). The scaling of critical piston amplitude with Reynolds number as $\overline{R e}^{-1 / 2}$, typical of boundary-layer damping, is clearly apparent in (5.10).

Figure 5 also shows the neutral curves if boundary-layer, rather than volumetric viscous, effects are neglected. The curves extend much lower in the $\left(\omega_{0}, \varepsilon\right)$-plane than they should, reflecting the relative smallness of the remaining volumetric damping mechanism for the low-order modes which yield the lowest values of $\varepsilon_{c}$. In summary, it is apparent that both volumetric and boundary-layer damping must be allowed for. The location of the lower boundary of the neutral curves in the $\left(\omega_{0}, \varepsilon\right)$-plane is mainly determined by boundary-layer damping, and thus scales roughly as $\overline{R e}^{-1 / 2}$, while volumetric damping stabilizes high-order mode pairs which would otherwise densely cover the $\left(\omega_{0}, \varepsilon\right)$-plane with unstable regions due to instability of shorter and shorter wavelengths. Note that, if viscosity were completely neglected, the entire region $\omega_{0}<2$ would be densely covered by unstable regions and the instability criterion would become simply $\omega_{0}<2$.

Figures $6(a)$ and $6(b)$ show the equivalent of figure 4 , but for different rotational Reynolds numbers, $\overline{R e}=5 \times 10^{3}$ and $\overline{R e}=2 \times 10^{4}$, respectively half and twice that of figure 4. Decreasing the Reynolds number tends to have a stabilizing effect, which is understandable given the role of viscosity as a damping mechanism, although 

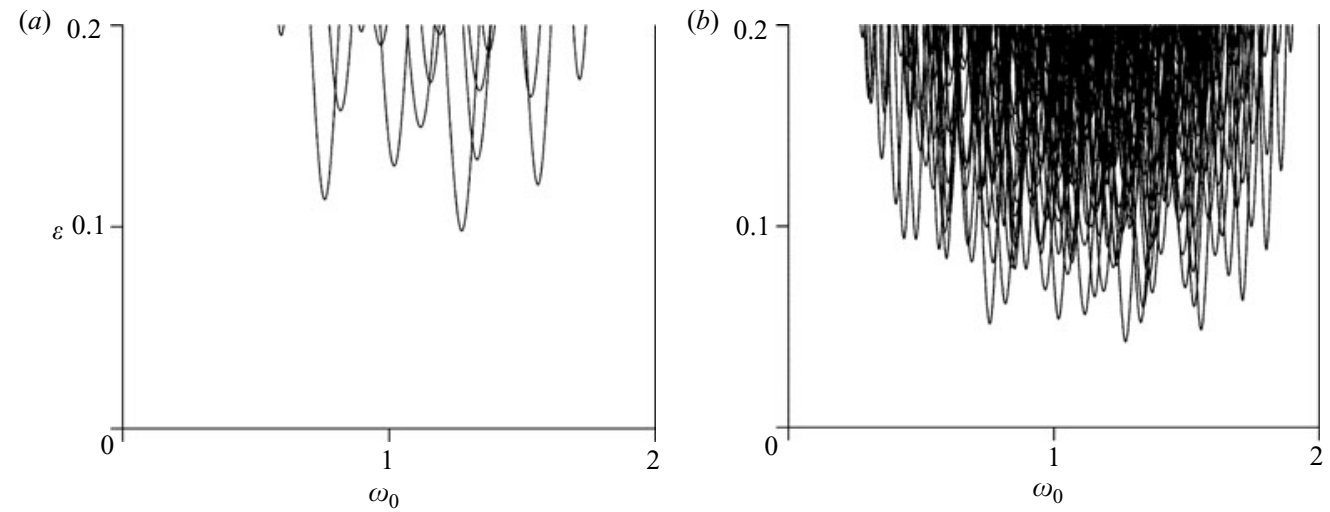

FIgURE 6. Neutral curves in the $\left(\omega_{0}, \varepsilon\right)$-plane for all modes with $(a) \overline{R e}=5 \times 10^{3}, h_{0}=2$, and (b) $\overline{R e}=2 \times 10^{4}, h_{0}=2$.

decreasing $\overline{R e}$ can destabilize individual mode pairs, as noted earlier. The neutral curves are denser at the higher Reynolds number, a reflection of the higher order of modes required to make volumetric damping important. In the limit, $\overline{R e} \rightarrow \infty$, the neutral curves reach down to $\varepsilon=0$, becoming straight-sided wedges with apex at $\omega_{0}=\omega_{+}-\omega_{-}$according to (5.8), wedges which fill the unstable region $\omega_{0}<2$. The same is true under inviscid theory and the overall instability criterion, $\omega_{0}<2$, does not depend on whether we take the limit $\overline{R e} \rightarrow \infty$ or consider the inviscid case from the start. However, as discussed earlier, the $\overline{R e} \rightarrow \infty$ limiting wedges do not in general coincide with their inviscid counterparts, so the details of precisely which mode pairs are unstable at given $\omega_{0}, \varepsilon$ differ depending on whether viscosity is included or not. As for the case without volumetric damping, denseness of the neutral curves at infinite Reynolds number reflects instability of an infinity of mode pairs with unboundedly small wavelengths, underlining the fact that $\overline{R e} \rightarrow \infty$ is a singular limit. Inviscid theory, while not agreeing with the $\overline{R e} \rightarrow \infty$ neutral curves, suffers from the same problem.

Note, from figures 4 and 6, that the Reynolds numbers involved are considerably larger than we might at first think, given the scaling $\overline{R e}=O\left(\varepsilon^{-2}\right)$. For instance, this scaling suggests that Reynolds numbers large compared with $10^{2}$ would be effectively infinite when $\varepsilon$ is of order 0.1 , whereas figure 4 , which is for $\overline{R e}=10^{4}$, indicates this is not the case, since the neutral curves of even the low-order modes are far from having taken on their infinite $\overline{R e}$, wedge-like forms. This is not to say that the scaling, $\overline{R e}=O\left(\varepsilon^{-2}\right)$, for significant viscous effects, is incorrect, but instead that there are nominally $O(1)$ numerical factors which are, in fact, rather far from 1 . Thus, viscous effects are considerably stronger than might be thought based on order-of-magnitude estimates.

Figures $7(a)$ and $7(b)$ show results for $\overline{R e}=10^{4}$ and two cylinder aspect ratios, $h_{0}=1$ and $h_{0}=10$. The former illustrates the stabilizing effect of taking smaller $h_{0}$. It should, however, be borne in mind that $\varepsilon$ is the piston amplitude divided by the cylinder height. Thus, if $h_{0}$ is reduced by decreasing the cylinder height at constant $\varepsilon$, the piston amplitude is reduced as well. Figure $7(b)$ illustrates the increasing density of neutral curves at large $h_{0}$. In the limit $h_{0} \rightarrow \infty$, the case of a cylinder of infinite length is approached. This infinite cylinder problem can be analysed in terms of waveguide modes for which the axial wavenumber $K_{Z}= \pm m \pi / h_{0}$ is a continuous 

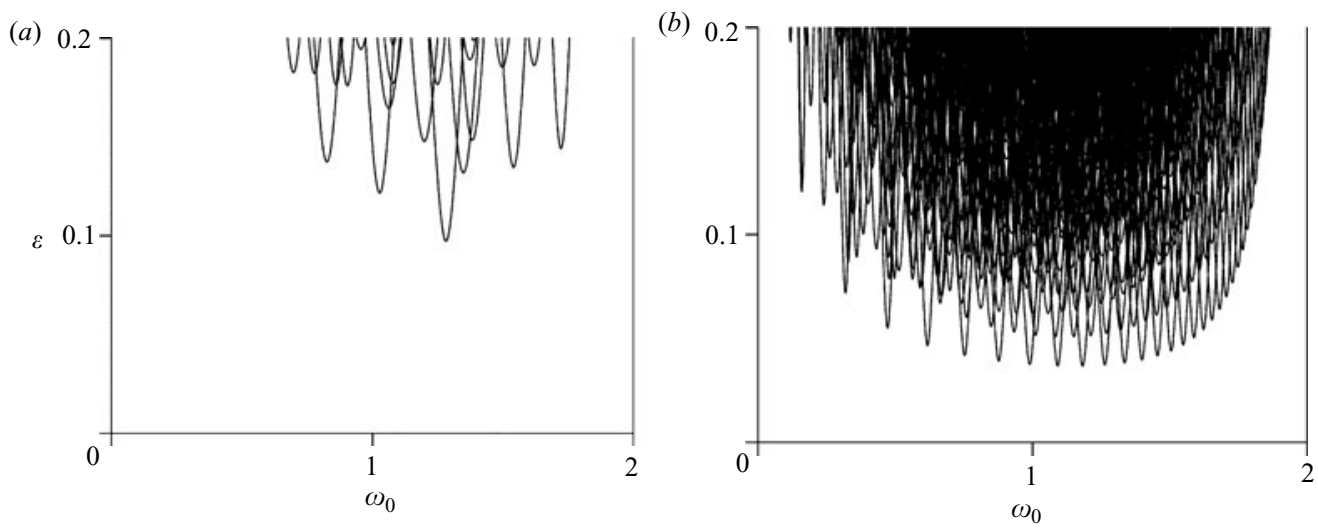

FIgURE 7. As figure 6, but with $(a) \overline{R e}=10^{4}, h_{0}=1$, and $(b) \overline{R e}=10^{4}, h_{0}=10$.

variable, a continuum which is the limit of more and more closely spaced, discrete axial wavenumbers and reflected in the high density of neutral curves of figure $7(b)$. However, it should be noted that the increasingly dense neutral curves as $h_{0} \rightarrow \infty$ do not represent smaller and smaller wavelengths, so this limit is not singular in the sense that $\overline{R e} \rightarrow \infty$ is.

\section{Conclusions}

This paper has concerned linear instability resulting from resonant interaction between small sinusoidal compression and the inertial-wave modes of a rotating cylinder, allowing for the stabilizing effects of viscous damping of both volumetric and boundary-layer origins, both of which are important. Beginning with the low-Machnumber formulation of the compressible Navier-Stokes equations and using adiabatic thermal boundary conditions, followed by transformation to a fixed cylinder geometry, the equations for the perturbation were derived and projected onto the inertial modes to yield equations for the modal amplitudes. Small-piston-amplitude asymptotics of these equations in $\S 4$ showed that, unless the cylinder aspect ratio has been specially tuned, attention may be restricted to pairs of modes whose frequency difference is close to the piston frequency. Analysis of the resulting mode-pair equations yields stability criteria which imply growth of a single axisymmetric inertial mode or a pair of non-axisymmetric ones above a piston amplitude, $\varepsilon$, which depends on $\omega_{0}$, $\overline{R e}$ and $h_{0}$ and whose graph in the $\left(\omega_{0}, \varepsilon\right)$-plane for fixed $\overline{R e}, h_{0}$ and a particular mode/mode pair gives the neutral hyperbola sketched in figure 3( $a)$. As illustrated by figures $2(b)$ and $2(c)$, axisymmetric modes consist of one or more oscillatory toroidal vortices encircling the cylinder axis, so the result of axisymmetric instability is the spontaneous growth of such a vortex system superimposed on the basic flow. The frequency of oscillation of the mode and hence of the vortex system is close to half that of the piston. The non-axisymmetric case is rather harder to visualize because of the presence of two growing modes of different frequencies and radial spatial structures, which interfere in a time-varying aperiodic fashion.

When all modes are superposed, we obtain the neutral hyperbolae plotted in figures 4, 6 and 7 for particular values of $\overline{R e}$ and $h_{0}$. Inside one of these neutral hyperbolae, the corresponding mode or mode pair is unstable, while in the interior of two or more neutral curves there are several growing modes/mode pairs. When there 
are multiple unstable mode pairs, we expect the fastest growing one to dominate at large times, at least in the linear regime considered here. The neutral curves depend on $\overline{R e}$ and $h_{0}$, tending to rise and become less dense when $\overline{R e}$ is reduced, reflecting the overall stabilizing effect of viscosity. Volumetric damping renders the density of neutral curves finite by suppression of short-wavelength high-order modes, while boundary-layer damping is the principal viscous mechanism for low-order ones, crucial in determining the critical piston amplitudes of the lower curves in the figures. Thus, both mechanisms are important in different ways, with boundary-layer damping being the main determinant of the minimum piston amplitude at which instability can occur for suitable choice of piston frequency.

In the case of the elliptic and triangular instabilities, Eloy et al. (2003) compared their experimental results with theoretical neutral curves allowing for both volumetric and boundary-layer damping and found good agreement, both in terms of the location of the stability boundary and the modes selected by the instability. Note that the amplitude equations, and hence the stability criterion, for these instabilities have the same form as for the present case, although the mode pairs they represent and the values of the coefficients are different. We expect the same to be generally true for instabilities of this kind. Experimental (Graftieaux et al. 2002) and DNS (Duguet et al. 2005) studies of the present instability for the lowest-order axisymmetric mode have shown good agreement with the theoretical neutral curve and also that the velocity field resulting from instability is very close to the inertial mode predicted by theory. In quantitative terms, the bottom point of the neutral curve in the $\left(\omega_{0}, \overline{R e}\right)$ plane determined using DNS with $h_{0}=1.18$ and $\varepsilon=0.131$ (the experimental values) differs from that of the present theory by just $3 \%$ in $\overline{R e}$ and $0.4 \%$ in $\omega_{0}$, while the corresponding experimental departures are $18 \%$ and $\sim 0.5 \%$.

Part 2 examines the fate of a linearly unstable mode or mode pair when it grows beyond the linear regime. Weakly nonlinear theory yields nonlinear mode-pair equations coupled to an infinite set of nonlinear amplitude equations for geostrophic modes of the family $n=m=0$.

J.-P. R. acknowledges support by the Service de la Recherche et des Etudes Amont for part of the study reported in this paper.

\section{Appendix A. Inertial-mode properties}

When $m \neq 0$ (non-geostrophic modes), the spatial eigenfunctions are

$$
\begin{gathered}
\chi_{r}^{(\mu)}=N^{(\mu)}\left(\omega^{(\mu)} k^{(\mu)} J_{n}^{\prime}\left(k^{(\mu)} r\right)-\frac{n}{r} J_{n}\left(k^{(\mu)} r\right)\right), \\
\chi_{\theta}^{(\mu)}=N^{(\mu)}\left(k^{(\mu)} J_{n}^{\prime}\left(k^{(\mu)} r\right)-\omega^{(\mu)} \frac{n}{r} J_{n}\left(k^{(\mu)} r\right)\right) \\
\chi_{Z}^{(\mu)}=N^{(\mu)} \frac{\omega^{(\mu)} k^{(\mu)^{2}} h_{0}}{m \pi} J_{n}\left(k^{(\mu)} r\right), \quad \Theta^{(\mu)}=N^{(\mu)}\left(1-\omega^{(\mu)^{2}}\right) J_{n}\left(k^{(\mu)} r\right)
\end{gathered}
$$

where $J_{n}$ and $J_{n}^{\prime}$ denote the Bessel function and its derivative and $k^{(\mu)}$ is a transverse wavenumber, one of the infinity of positive real roots of

$$
k^{(\mu)} J_{n}^{\prime}\left(k^{(\mu)}\right)= \pm n\left(1+\left(\frac{k^{(\mu)} h_{0}}{m \pi}\right)^{2}\right)^{1 / 2} J_{n}\left(k^{(\mu)}\right),
$$


each of which yields a mode (or pair of conjugate modes if $n=0$ ) of the family $n, m$. The modal frequency is determined by

$$
\omega^{(\mu)}= \pm\left(1+\left(\frac{k^{(\mu)} h_{0}}{m \pi}\right)^{2}\right)^{-1 / 2},
$$

where the sign should be chosen as in (A 2). The choice of signs in (A 2) and (A 3) leads to a subdivision of the modal family $n, m$ into positive and negative frequencies in $\left|\omega^{(\mu)}\right|<1$. The normalization constant $N^{(\mu)}$ in (A 1$)$ is

$$
N^{(\mu)}=\left(\pi h_{0} \eta^{(\mu)}\right)^{-1 / 2} \frac{m \pi}{h_{0} k^{(\mu)} J_{n}\left(k^{(\mu)}\right)},
$$

where $\eta^{(\mu)}=n \omega^{(\mu)}+n^{2}+m^{2} \pi^{2} / h_{0}^{2}$. Equation (A 4) results from requiring that (3.6) holds when $v=\mu$, determining the volume integral using (3.4), (A 1)-(A 3), and the evaluation of integrals of trigonometric and Bessel functions.

Numerical implementation of the stability analysis of this paper requires the positive roots of (A 2). The positive zeros of $J_{n}$ and $J_{n}^{\prime}$ may first be determined by stepping upwards in multiples of $\pi$ from 0 to find intervals in which there is a change of sign, followed by interval halving to compute the zeros to machine precision. If $n=0$, the zeros of $J_{n}^{\prime}$ yield the $k^{(\mu)}$ directly. When $n \neq 0$, it can be shown that the positive roots of (A 2) are interlaced with the positive zeros of $J_{n}$ and $J_{n}^{\prime}$, so interval halving can again be used to determine the $k^{(\mu)}$. The Bessel function and its derivative are evaluated using a high-precision standard library routine.

For geostrophic modes $\left(m=0, \omega^{(\mu)}=0\right)$,

$$
\begin{gathered}
\chi_{r}^{(\mu)}=\frac{n N^{(\mu)}}{r}\left(J_{n}\left(k^{(\mu)}\right) r^{|n|}-J_{n}\left(k^{(\mu)} r\right)\right), \quad \chi_{\theta}^{(\mu)}=N^{(\mu)}\left(k^{(\mu)} J_{n}^{\prime}\left(k^{(\mu)} r\right)-|n| J_{n}\left(k^{(\mu)}\right) r^{|n|-1}\right), \\
\chi_{Z}^{(\mu)}=0, \quad \Theta^{(\mu)}=N^{(\mu)}\left(J_{n}\left(k^{(\mu)} r\right)-J_{n}\left(k^{(\mu)}\right) r^{|n|}\right),
\end{gathered}
$$

where $k^{(\mu)}$ is one of the infinite set of positive real roots of

$$
k^{(\mu)} J_{n}^{\prime}\left(k^{(\mu)}\right)=|n| J_{n}\left(k^{(\mu)}\right),
$$

each of which yields a different geostrophic mode of azimuthal order $n$. From (A 5) and (A 6), $\boldsymbol{u}^{(\mu)}=0$ on $r=1$, i.e. the no-slip condition is satisfied on the sidewalls. The normalization constant in (A 5) is

$$
N^{(\mu)}=\left(\pi h_{0}\right)^{-1 / 2}\left(k^{(\mu)} J_{n}\left(k^{(\mu)}\right)\right)^{-1} .
$$

Whether or not they are geostrophic, it is apparent from (A 2) and (A 6) that axisymmetric modes derive from the positive roots of $J_{0}^{\prime}\left(k^{(\mu)}\right)=0$.

From (3.4), (A 1) and (A 5), it can be shown that

$$
\nabla^{2} \boldsymbol{u}^{(\mu)}+K^{(\mu)^{2}} \boldsymbol{u}^{(\mu)}=\nabla \phi^{(\mu)}
$$

for all modes, where $\phi^{(\mu)}$ is zero for non-geostrophic modes and

$$
\phi^{(\mu)}=\mathrm{i} \operatorname{sgn}(n) N^{(\mu)} k^{(\mu)^{2}} J_{n}\left(k^{(\mu)}\right) r^{|n|} \mathrm{e}^{\mathrm{i} n \theta}
$$

when $\mu$ is geostrophic.

Finally, the coupling matrix $C_{\mu \nu}=\int u_{Z}^{(\mu)^{*}} u_{Z}^{(\nu)} \mathrm{d}^{3} \boldsymbol{X}$ can be determined using (3.4), (A 1)-(A 3) and the evaluation of integrals of trigonometric and Bessel functions. Aside from the properties given in the text at the end of $\S 3$, when $m>0$ we find

$$
C_{\mu \mu}=-C_{\mu \mu^{*}}=\frac{1}{2}\left(1-\omega^{(\mu)^{2}}\right)
$$


for axisymmetric modes and

$$
\begin{aligned}
& C_{\mu \mu}=\frac{1}{2}\left(1-\omega^{(\mu)^{2}}\right) \frac{\eta^{(\mu)}-n \omega^{(\mu)}}{\eta^{(\mu)}}, \\
& C_{\mu \nu}=\frac{n k^{(\mu)} k^{(\nu)}\left(\omega^{(\mu)}-\omega^{(\nu)}\right)}{\left(\eta^{(\mu)} \eta^{(\nu)}\right)^{1 / 2}\left(k^{(\mu)^{2}}-k^{(\nu)^{2}}\right)}, \quad \mu \neq v,
\end{aligned}
$$

for modes $\mu, v$ of the same non-axisymmetric family. Equations (A 10) for $n=0$, $m>0$ and (A 11), (A 12) for $\mu, v$ belonging to the same $n \neq 0, m>0$ family yield all non-zero $C_{\mu \nu}$.

\section{Appendix B. Boundary-layer analysis}

The object of this Appendix is the derivation of (4.6) using leading-order boundarylayer analysis. Consider a boundary layer away from the corners of the cylinder. As in classical boundary-layer theory, (2.17) and zero normal velocity at the wall imply that $\boldsymbol{u}$ is parallel to the wall at leading order, while the normal component of (2.18) implies that the pressure variable $\lambda$ does not vary significantly across the layer, being imposed from outside. Taking the component of (2.18) tangential to the wall, the fact that $\boldsymbol{u}$ is dominantly tangential allows approximation of the Coriolis term on the left by $\boldsymbol{e} \times \boldsymbol{u}$ at leading order, where $\boldsymbol{e}=\left(\boldsymbol{e}_{Z} \cdot \boldsymbol{n}\right) \boldsymbol{n}$ is the projection of $\boldsymbol{e}_{Z}$ normal to the wall. All terms on the right-hand side of the tangential component of (2.18) are negligible at leading order, apart from the viscous term, which is promoted to leading order by thinness of the layer and can be approximated as $\partial^{2} \boldsymbol{u} / \partial \xi^{2}$, where $\overline{R e} \bar{R}^{-1 / 2} \xi$ is distance from the wall. Thus, one obtains the leading-order boundary-layer equation for the perturbation flow

$$
\frac{\partial \boldsymbol{u}}{\partial t}+\boldsymbol{e} \times \boldsymbol{u}-\frac{\partial^{2} \boldsymbol{u}}{\partial \xi^{2}}=-\nabla_{\|} \lambda,
$$

where $\boldsymbol{u}$ is parallel to the wall and $\nabla_{\|} \lambda$ is the perturbation pressure gradient parallel to the wall, independent of $\xi$ and imposed from outside the layer. The boundary conditions to go with (B 1) are that $\boldsymbol{u}=0$ at $\xi=0$ and $\boldsymbol{u} \rightarrow \boldsymbol{u}_{\infty}$ as $\xi \rightarrow \infty$, where $\boldsymbol{u}_{\infty}$ is the leading-order external flow, extrapolated to the wall. Since viscosity is unimportant outside the boundary layer, $\nabla_{\|} \lambda$ and $\boldsymbol{u}_{\infty}$ are related by (B 1) without the viscous term.

Outside the boundary layer, the leading-order flow is a combination of inertial modes with amplitudes $\delta A_{\mu}$ and frequencies $\omega^{(\mu)}$. Each such mode forces motion in the boundary-layer via its contributions to the right-hand side of (B1) and to $\boldsymbol{u}_{\infty}$, while linearity of (B 1) implies that the response of the layer is a sum over the modal contributions taken separately. With unit modal amplitude, the response induced by mode $v$ is obtained by solving (B 1) with $-\nabla_{\|} \lambda^{(v)} \exp \left(-\mathrm{i} \omega^{(v)} t\right)$ on the right-hand side and the boundary conditions $\boldsymbol{u}=0$ at $\xi=0$ and $\boldsymbol{u} \rightarrow \boldsymbol{u}^{(\nu)} \exp \left(-\mathrm{i} \omega^{(\nu)} t\right)$ as $\xi \rightarrow \infty$, where $\nabla_{\|} \lambda^{(v)}$ and $\boldsymbol{u}^{(v)}$ are to be evaluated at the wall. Following a transient period of duration $O(1)$, which does not concern us here since we are interested in the evolution at longer times, the solution for $\boldsymbol{u}$ takes on the same time dependence as the forcing, namely $\exp \left(-\mathrm{i} \omega^{(v)} t\right)$. For the side-wall layer, $\boldsymbol{e}=0$ (i.e. rotation is unimportant) and the velocity has the classical Stokes-layer form

$$
\boldsymbol{u}=\boldsymbol{u}^{(v)} \exp \left(-\mathrm{i} \omega^{(v)} t\right)\left(1-\exp \left(-\left(-\mathrm{i} \omega^{(v)}\right)^{1 / 2} \xi\right)\right)
$$


whereas for the end-wall boundary layers $\boldsymbol{e}=\boldsymbol{e}_{Z}$ and the velocity has the Ekman-layer form

$$
\begin{aligned}
\boldsymbol{u}= & \exp \left(-\mathrm{i} \omega^{(v)} t\right)\left\{\boldsymbol{u}^{(v)}-\frac{1}{2}\left(\left(\boldsymbol{u}^{(v)}+\mathrm{i} \boldsymbol{e}_{Z} \times \boldsymbol{u}^{(v)}\right) \exp \left(-\gamma_{+}^{(v)} \xi\right)\right.\right. \\
& \left.\left.+\left(\boldsymbol{u}^{(v)}-\mathrm{i} \boldsymbol{e}_{Z} \times \boldsymbol{u}^{(v)}\right) \exp \left(-\gamma_{-}^{(v)} \xi\right)\right)\right\}
\end{aligned}
$$

where

$$
\gamma_{ \pm}^{(v)}=\left(-\mathrm{i}\left(\omega^{(v)} \pm 1\right)\right)^{1 / 2}
$$

and principal values are taken for the complex square roots in (B2) and (B 4), in order that the exponential functions of $\xi$ decay to zero, rather than growing, as $\xi \rightarrow \infty$.

We may calculate the normal derivative $\boldsymbol{n} \cdot \nabla \boldsymbol{u}=-\overline{R e}^{1 / 2} \partial \boldsymbol{u} / \partial \xi$ at the wall $\xi=0$ from (B 2) or (B 3) and hence determine the leading-order contribution of mode $v$ (with unit amplitude) to the surface integral in (4.6). Multiplying the result by $\delta A_{v}$ and summing over $v$ gives (4.6) with

$$
\begin{aligned}
D_{\mu \nu}= & \underbrace{\left(-\mathrm{i} \omega^{(\nu)}\right)^{1 / 2} \int_{r=1} \boldsymbol{u}^{(\mu)^{*}} \cdot \boldsymbol{u}^{(\nu)} \mathrm{d}^{2} \boldsymbol{X}}_{\text {Sidewall boundary layer }} \\
& +\underbrace{\frac{1}{2}\left(\gamma_{+}^{(v)}+\gamma_{-}^{(v)}\right) \int_{Z=0, h_{0}} \boldsymbol{u}^{(\mu)^{*}} \cdot \boldsymbol{u}^{(\nu)} \mathrm{d}^{2} \boldsymbol{X}+\frac{1}{2} \mathrm{i}\left(\gamma_{+}^{(v)}-\gamma_{-}^{(v)}\right) \int_{Z=0, h_{0}} \boldsymbol{u}^{(\mu)^{*}} \cdot\left(\boldsymbol{e}_{Z} \times \boldsymbol{u}^{(v)}\right) \mathrm{d}^{2} \boldsymbol{X}}_{\text {Endwall boundary layers }}
\end{aligned}
$$

Note that, in deriving (B 5), departures from (B 2) and (B 3) near the cylinder corners have been neglected on the grounds that the corner regions are small and hence contribute little to the surface integral. It can easily be shown using (3.4) that $D_{\mu \nu}$ is zero unless $n_{\mu}=n_{v}$. It can also be shown that $D_{\mu \nu}=0$ if $\mu \neq v$ are both geostrophic modes, i.e. there is no viscous coupling between such modes, a result which will be used in Part 2.

It is possible to evaluate (B 5) using (3.4) and the expressions for the modal eigenfuctions of Appendix A, but the results are very lengthy and are not given here. However, the diagonal components of $D_{\mu \nu}$ are required in deriving the results of $\S 5.2$ and are given by

$$
\begin{aligned}
D_{\mu \mu}= & \mathrm{i} \frac{\left(1-\omega^{(\mu)^{2}}\right)^{1 / 2}}{\eta^{(\mu)} h_{0}}\left\{\left(\eta^{(\mu)}+n \omega^{(\mu)}\right)\left(\gamma_{+}^{(\mu)}-\gamma_{-}^{(\mu)}\right)\right. \\
& \left.+\left(\eta^{(\mu)}-n \omega^{(\mu)}\right)\left(\omega^{(\mu)}\left(\gamma_{+}^{(\mu)}+\gamma_{-}^{(\mu)}\right)-\mathrm{i} h_{0}\left(1-\omega^{(\mu)^{2}}\right)^{1 / 2}\left(-\mathrm{i} \omega^{(\mu)}\right)^{1 / 2}\right)\right\}
\end{aligned}
$$

for non-geostrophic modes and

$$
D_{\mu \mu}=\frac{2^{1 / 2}}{h_{0}}
$$

for geostrophic $\mu$. Equation (B 7) does not depend on which geostrophic mode is considered, implying the same viscous decay rate for all geostrophic modes if volumetric damping is neglected. 


\section{REFERENCES}

Aldridge, K. D. \& Toomre, A. 1969 Axisymmetric inertial oscillations of a fluid in a rotating spherical container. J. Fluid Mech. 37, 307-323.

BAINEs, P. G. 1967 Forced oscillations of an enclosed rotating fluid. J. Fluid Mech. 30, 533-546.

Duguet, Y., Scott, J. \& Le Penven, L. 2005 Instability inside a rotating gas cylinder subject to axial periodic strain. Phys. Fluids 17, 114103.

Eloy, C. \& Le Dizés, S. 2001 Stability of the Rankine vortex in a multipolar strain field. Phys. Fluids 13, 660-676.

Eloy, C., Le GaL, P. \& Le DizÉs, S. 2003 Elliptic and triangular instabilities in rotating cylinders. J. Fluid Mech. 476, 357-388.

Fultz, D. 1959 A note on overstability, and the elastoid-inertia oscillations of Kelvin, Solberg and Bjerknes. J. Met. 16, 199-208.

Gans, R. F. 1970 On the precession of a resonant cylinder. J. Fluid Mech. 41, 865-872.

Gledzer, E. B. \& Ponomarev, V. M. 1992 Instability of bounded flows with elliptical streamlines. J. Fluid Mech. 240, 1-30.

Graftieaux, L., Le Penven, L., Scott, J. F. \& Grosjean, N. 2002 A new parametric instability in rotating cylinder flow. In Advances in Turbulence IX (ed. I. P. Castro \& P. E. Hancock). CIMNE, Barcelona, Spain.

Greenspan, H. P. 1969 The Theory of Rotating Fluids. Cambridge University Press.

KeLvin, LoRd 1880 Vibrations of a columnar vortex. Phil. Mag. 10, 155-168.

Kerswell, R. R. 1993 The instability of precessing flow. Geophys. Astrophys. Fluid Dyn. 72, 107-144.

Kerswell, R. R. 1999 Secondary instabilities in rapidly rotating fluids: inertial wave breakdown. J. Fluid Mech. 382, 283-306.

Kerswell, R. R. 2002 Elliptical instability. Annu. Rev. Fluid Mech. 34, 83-114.

Kerswell, R. R. \& Barenghi, C. F. 1994 On the viscous decay of inertial waves in a rotating circular cylinder. J. Fluid Mech. 285, 203-214.

Kobine, J. J. 1995 Inertial wave dynamics in a rotating and precessing cylinder. J. Fluid Mech. 303, $233-252$.

KUDLICK, M. 1966 On the transient motions in a contained rotating fluid. PhD thesis, MIT.

Le Noble, J. 1995 Etude de l'écoulement d'un fluide dans un cylindre en rotation uniforme autours de son axe. DEA dissertation, Laboratoire de Mécanique des Fluides et d'Acoustique, Ecole Centrale de Lyon, Ecully, France.

McEwan, A. D. 1970 Inertial oscillations in a rotating fluid cylinder. J. Fluid Mech. 40, 603-640.

Mahalov, A. 1993 The instability of rotating fluid columns subjected to a weak external Coriolis force. Phys. Fluids A 5(4), 891-900.

MannaseH, R. 1994 Distortions of inertia waves in a rotating fluid cylinder forced near its fundamental mode resonance. J. Fluid Mech. 265, 345-370.

Mansour, N. N. \& Lundgren, T. S. 1990 Three-dimensional instability of rotating flows with oscillating axial strain. Phys. Fluids A 2, 2089-2091.

RaCZ, J.-P. \& SCOTT, J. F. 2008 Parametric instability in a rotating cylinder of gas subject to sinusoidal axial compression. Part 2. Weakly nonlinear theory. J. Fluid Mech. 595, 291-321.

RIEUTORD, M. 1991 Linear theory of rotating fluids using spherical harmonics. Part II, time-periodic flows. Geophys. Astrophys. Fluid Dyn. 59, 185-208.

Stergiopoulos, S. \& Aldridge, K. D. 1982 Inertial waves in a fluid partially filled cylindrical cavity during spin-up from rest. Geophys. Astrophys. Fluid Dyn. 21, 89-112.

WALEFFE, F. 1990 On the three-dimensional instability of strained vortices. Phys. Fluids A 2, 76-80. 